\title{
Monumentos, nudos en el pañuelo. Megalitos, nudos en el espacio: Análisis del emplazamiento de los monumentos tumulares gallegos
}

\author{
Felipe Criado Boado y Jacobo Vaquero Lastres *
}

"También podriamos citar aqui los signos memorativos, como el famoso nudo en el pañuelo, los monumentos...». Husserl, Investigaciones lógicas.

\section{PRESENTACIÓN}

Intención. Se trata de definir las circunstancias que subyacen al emplazamiento de los túmulos o "mámoas" gallegas, tanto con estructuras megalíticas como sin ellas. Se intenta mostrar cómo la situación de esos monumentos presenta una fuerte uniformidad que, desde nuestro punto de vista, señalaría que la elección del lugar de construcción de los mismos no fue dejada al azar, sino que, antes bien, bajo ella subyace una profunda racionalidad. Observando las recurrencias que se manifiestan en el emplazamiento tumular, se pretende evidenciar el proceso al que responde la selección del punto concreto de levantamiento de una estructura monumental.

Alineación. Este trabajo prolonga estudios anteriores sobre el Megalitismo del NW peninsular centrados en torno al reconocimiento fundamental de que a través del megalitismo se opera una construcción específica del paisaje social dentro de la cual emergen conceptos innovadores del espacio y tiempo. Creemos que el megalitismo, como realidad cultural, produce una determinada experiencia simbólico-ritual del espaciotiempo social que es coherente con las racionalidades económicas y los

* Dept. de Historia 1, Fac. Xeog. e Historia. Univ. de Santiago. SANTIAGO. 
procesos sociales que marcan la transformación desde las primeras y sencillas comunidades neolíticas, hacia las sociedades campesinas complejas marcadas por el surgimiento de la división y de todo el entramado sociocultural que acompaña a ésta ${ }^{1}$.

Cautela. A pesar de que nuestro objetivo esencial no es producir explicaciones o predicciones en sentido estricto, ofrecemos al final del trabajo una posible "contrastación" de estas interpretaciones. Con todo, creemos que la positividad de nuestras propuestas viene dada por el hecho de que, a través de ellas, se pueden describir e interpretar fenómenos y circunstancias que, de otro modo, resultaban invisibles o incomprensibles.

Fundamentos. Los autores reúnen entre los dos una amplia experiencia en la prospección sistemática de túmulos: en los últimos diez años han catalogado alrededor de 1.200 mámoas y prospectado unos 1.000 $\mathrm{km}^{2}$ de superficie. En toda esa labor han orientado sus pasos guiados por preguntas que se referían al emplazamiento, a la posición y a la visibilidad de los túmulos en el paisaje, y por la continua sorpresa de que existan mámoas en unas zonas y no en cambio en otras aparentemente semejantes ${ }^{2}$.

Limitaciones. Toda esa información no ha sido objetivada de acuerdo con herramientas estadísticas o informáticas que pudieran aportar una mayor fidelidad en el tratamiento de la documentación. La sistematización se ha basado siempre en la comprensión etnográfica del paisaje observado, en el análisis cartográfico directo y en el tratamiento de las condiciones topográficas a través de técnicas de dibujo lineal.

Perspectivas. Este trabajo describe el modelo de emplazamiento tumular que, desde nuestro punto de vista, surge a partir de una elaboración preliminar de la información disponible. Pero esa descripción no es más que una anticipación que deberá orientar trabajos futuros y ser contrastada ante sus resultados. En este sentido, el presente texto ofrece los planteamientos iniciales que subyacen a un proyecto de estudio del emplazamiento y ritual tumular en Galicia actualmente en curso de realización.

indice. En primer lugar se revisará el interés que posee estudiar el emplazamiento megalítico criticando algunas de las consideraciones tra-

Ver detalles en CRIADO BOAdO 1989a; sobre el concepto "división" y estas posiciones véase P. Clastres 1981.

2 En este sentido nos basaremos en análisis anteriores recogidos en la bibliografía (VAQuero 1989a, 1990b y 1990c, Criado $1984-5$ y Criado et all. 1986). 
Monumentos, nudos en el pañuelo. Megalitos, nudos en el espacio: Análisis...

dicionales sobre este tema (apartado 2.1), al tiempo que se precisarán conceptos y principios que utilizaremos en este trabajo (apartado 2.2); a continuación se analizarán todos los elementos implicados en el proceso de emplazamiento tumular estableciendo una distinción que creemos útil (apartado 3 y subapartados sucesivos); por último terminaremos con una sintesis (apartado 4).

\section{INTRODUCCIÓN: LA ARQUITECTURA DEL EMPLAZAMIENTO}

El principal elenco de datos que posee el investigador del fenómeno tumular en Galicia viene dado por el mapa de situación de los monumentos existentes en una determinada zona. Ese mapa, en la medida en que es el producto de procesos sociales, podría informar sobre éstos y sobre las comunidades que los protagonizaron. Sin embargo, el predominio en nuestra disciplina de una voluntad de saber empirista y cronológica conduce en cierta medida a despreciar esa información en aras de los datos, aparentemente más sustanciales, aportados por la excavación, el análisis tipológico (de las estructuras o de las piezas de ajuar) y las determinaciones paleo-ecológicas.

Esta pre-selección del registro a considerar viene dada en gran medida por la ausencia de un aparato conceptual adecuado para examinar ese tipo de realidades. Al no existir éste, resulta difícil extraer consecuencias adecuadas de ese tipo de datos y se encuentran pronunciamientos que alegan que del estudio del emplazamiento megalítico no se puede derivar ninguna conclusión importante sobre las sociedades constructoras:

«O arqueólogo observa centenares de mamoas..., Podemos estudar a relaÇao dessses monumentos com a cha, com o méio-ambiente, mas é imposível sen uma escavaÇao saber qual é o mais antigo ou o mais recente. Por tanto é un pouco falivel o estudo destes monumentos en termos de espaÇo, porque nao é possível definir previamente a que época é que pertencen..." (Cruz 1988: 51) ${ }^{3}$.

En este sentido, el propósito del presente apartado es establecer las categorías, principios y conceptos fundamentales con los que se podría invertir esa situación e iniciar un análisis rentable del emplazamiento megalítico. Para ello observaremos en 2.1 las categorías básicas de partida

\footnotetext{
3 Esta situación ejemplifica la hegemonia que el tiempo ha ejercido dentro de las disciplinas históricas y que ha dificultado la comprensión del espacio; sobre estos temas y su influencia en la Arqueología del Espacio se puede ver CaIADO BOADO 1991
} 
y revisaremos algunos tópicos sobre el emplazamiento, y en 2.2 definiremos la estrategia concreta que nosotros seguimos.

\subsection{Asentamiento y emplazamiento: las vicisitudes de la geología}

Desde un primer momento se hizo patente que el estudio de la situación de las mámoas gallegas se podía realizar a dos niveles: general (a escala de una comarca determinada) o particular (a escala de un conjunto concreto de monumentos). Tamaña división es obvia y en parte sigue la diferenciación entre niveles macro y semi-macro de CLARKE 1977. Sin embargo, el interés de esta dualidad de perspectivas radica en que, a través de ella, nos aproximábamos a dos hechos arqueológicos muy distintos, a dos aspectos relativamente independientes de las sociedades megaliticas.

Por el primer camino nos era dado observar la "distribución de túmulos" en su conjunto que, en líneas generales, debería estar en relación con el asentamiento de las comunidades megalíticas y nos podría ilustrar sobre las condiciones de carácter tecno-economo-ambiental que influían en aquella ${ }^{4}$. Por el segundo camino nos aproximábamos, en cambio, al «emplazamiento de los monumentos" que, por su parte, estaría más relacionado con factores de carácter ritual y simbólico-imaginario que con otro tipo de circunstancias (CRIADo et all 1986:129). El estudio de esta segunda temática es muy rentable, aunque presenta diversas dificultades.

En efecto, como consecuencia de lo que podemos denominar la cinfraestructura geológica" del megalitismo (el hecho de que el sustrato sobre el que se emplazan los monumentos [u otro distinto] haya sido utilizado para construir las cámaras), durante mucho tiempo el megalitismo en general y las aproximaciones espaciales al fenómeno, en particular, han sido herederas de una tradición inconsciente que llevó a sobredimensionar el efecto del medio geológico sobre la distribución megalíti$\mathrm{ca}^{5}$.

Así surge la noción de que la causalidad fundamental del emplazamiento megalítico se encontraría en la disponibilidad de material geológico adecuado en las proximidades del monumento para construir la cámara.

\footnotetext{
4 Respecto a Galicia véase CrIAdo et all. 1986 y CRIADO BoAdo 1989b y 1988; para otras regiones Carlos IzQUIERdo 1988 o en general ChAPMAN 1987.

5 En Galicia esta tradición arranca de G. LEISNER 1938; en otras zonas véase por ejemplo L'HELGOUACH 1965.
} 
Cuando se racionalizaba o culturalizaba este condicionante, se concluía asimismo que él, por su parte, reflejaba que la causa determinante de la elección del punto de levantamiento de un monumento sería la capacidad de movilización de mano de obra por parte de una comunidad concreta para trabajar en su monumento, ya que la cantidad de gente de la que se dispusiera implicaria que el monumento podría estar más de lejos de las fuentes de extracción de la materia prima o debería estar forzosamente ceñido a las mismas.

Este tipo de categorización imposibilitó durante bastante tiempo realizar una aproximación al emplazamiento que contemplase a éste más como el efecto de procesos culturales complejos (fundamentalmente implicando elementos de orden ritual y simbólico), que como el reflejo directo de presuntas condiciones de existencias de las sociedades megalíticas. En este contexto el papel simbólico del monumento quedó encerrado durante bastante tiempo en una mera apelación al megalito como un símbolo externo ajeno a la realidad social de la comunidad que lo construyó y en la que el megalito entendido como símbolo se concretaba en la figura simplista de un monumento comprendido como mojón territorial ${ }^{6}$.

Dejando a un lado otras consideraciones sobre el tema de la geología (volveremos sobre ellas más abajo), interesa destacar que detrás de estos posicionamientos emerge un tipo de análisis muy simplista que se basa en el establecimiento de relaciones directas entre el proceso que se estudia (el emplazamiento) y los elementos materiales asociados a los datos que se consideran (la situación de los túmulos). Esta inducción hipersimplificadora caracteriza en general al funcionalismo y al empirismo, tanto en sus versiones elaboradas y "científicas" (que representa la New Archaeology), como en las versiones cotidianas y mundanas que constituyen la "lógica del sentido común" con la que mayormente se afronta la vida diaria y que afloran de un modo natural o inconsciente en la observación científica no rigurosa ni auto-limitada por una adecuada perspectiva teórica ${ }^{7}$.

Este tipo de hiper-simplificación funcionalista es la misma que establece una relación directa entre emplazamiento, geología, fuerza de tra-

Es la posición de Fleming 1973 y Renfrew 1976, todavía defendida en Renfrew 1987.

Tal y como, por ejemplo, argüye CHALMERS (1989: 197); "El falso supuesto de que hay un método científico universal al que deberían ajustarse todas las formas de conocimiento desempeña un papel perjudicial en nuestra sociedad, especialmente a la luz del hecho de que la versión del método científico a la que normalmente se recurre es una tosca versión empirista o inductivista». 
bajo y capacidad demográfica de la sociedad constructora ${ }^{8}$. Reaparece asimismo en el argumento que vincula los túmulos a vías de comunicación haciendo aparecer a esa relación como una característica más o menos recurrente del emplazamiento megalítico y que nuevamente consiste en asociar de forma directa los monumentos a elementos materiales concretos (en este caso caminos). Tal y como se verá más tarde ${ }^{9}$, este "relacionismo de orden directo" oscurece una aproximación más rentable al tema de la vinculación entre megalitos y tránsito que, como tal, es un fenómeno cultural de naturaleza previa y relativamente independiente de los elementos físicos (caminos) en los que se puede llegar a concretar.

Estas consideraciones, además de servirnos en sentido concreto para matizar dos circunstancias que tradicionalmente se han reseñado como elementos esenciales del emplazamiento tumular, nos sirven sobre todo para alcanzar un principio de orden teórico más general. Pues resulta que nuestra "mirada» al fenómeno, en vez de fijarse en las circunstancias físicas y materiales directa e inmediatamente asociadas a los monumentos, deberá mirar a través de ellas para centrarse en el proceso cultural que ellas concretan. Esta estrategia «racionalista» es un principio básico de nuestro trabajo.

\subsection{Disposición y vinculación: la racionalidad de la arquitectura}

Entre todas las estrategias legítimas de trabajo que se pudieran definir respecto al fenómeno tumular del NW, la línea por la que optaron los presentes autores consistió en intensificar en primer lugar los trabajos sistemáticos de prospección para, de este modo, configurar una visión lo más completa posible del "espacio extra-tumular», dejando para un momento ulterior el inicio de las labores de excavación en área que permitieran examinar la situación intra-tumular ${ }^{10}$.

- Esta sobre-simplificación se percibe bien en las reconstrucciones funcionalistas de los 70 de las comunidades constructoras de monumentos megalíticos (nota 6). BELLo DiEGUEz et all. 1984 reproduce una posición parecida, aún cuando en este caso lo que subyace es la utilización de la cantidad mínima de fuerza de trabajo que requirieron diferentes monumentos como un indicio indirecto de complejidad lo cual, pese a los problemas teóricos que presenta, puede ser aceptado en cierta medida

9 Y como se vio en otro trabajo: VAQUERo LASTRES $1990 \mathrm{~b}$.

10 Estos trabajos se han iniciado de hecho desde 1988, fundamentalmente en el marco de las campañas de trabajo de campo del Proyecto de Arqueología del Paisaje realizado en la Sierra de O Bocelo y en el valle del rio Furelos, con financiación de la Consellería de Cultura de la Xunta de Galicia; en este contexto los autores han codirigido la excavación de tres túmulos de diferentes tipos entre 1988 y 1989, (véase CrIado BoAdo y VaQuero Lastres 1990). Durante los últimos dos años (1990 y 1991) estas excavaciones se han intensificado en el marco del 
De este modo, nuestra estrategia de trabajo plantea un análisis tipo zoom a través de todos los niveles o escalas que constituyen la espacialidad megalítica. En este sentido se basa en una serie de presupuestos teórico-interpretativos descritos en trabajos previos.

El primero de esos presupuestos se basa en reconocer que el megalitismo debe ser entendido ante todo como un fenómeno esencialmente "espacial». A fin de cuentas, en la medida en que el megalitismo es un tipo (el primero) de "arquitectura monumental», es obvio que su dimensión fundamental es de carácter espacial. Creemos que es de esta circunstancia de donde se deben extraer las categorías de análisis y los modelos interpretativos básicos para tratar con ese fenómeno cultural.

De este modo, nuestro presupuestoo plantea que la monumentalidad megalítica se construye a través de un proceso complejo que supone la interacción y conjugación de toda una amplia serie de niveles espaciales que se escalonan desde el interior de la cámara y el patrón de distribución de los elementos denominados de "ajuar", hasta el exterior del túmulo y sus estructuras de entrada.

Ahora bien, considerada la monumentalidad megalítica desde este punto de vista, nuestro tercer presupuesto es que el nivel primario de arquitecturación del megalitismo es el emplazamiento de los túmulos. Este no es sólo un dato más a tener en cuenta, sino que desde nuestra perspectiva sería el primer recurso en base al cual se construye el espacio megalíti$\mathrm{co}^{11}$.

Para seguir adelante con este estudio sobre el emplazamiento es necesario definir los conceptos que utilizaremos. Llamaremos «localización" al punto concreto de situación de un túmulo. $Y$ lo diferenciaremos de lo que llamaremos "disposición", término con el que nos referiremos a la situación de ese túmulo en relación con elementos físicos y materiales asociados de forma directa e inmediata con él. Esos elementos son los que se perciben con la mera observación empírica al constatar que, dada la localización de una mámoa concreta, ésta "se dispone» al lado de tal elemento o tal otro.

Proyecto Arqueológico Endesa-As Pontes que, dirigido por JVL, lleva a cabo a través de un Convenio firmado entre la Consellería de Cultura y la empresa ENDESA la excavación de todos los túmulos afectados por la explotación de lignito a cielo abierto de As Pontes de García Rodríguez (Coruña), habiéndose excavado hasta el momento siete monumentos diferentes.

Estos presupuestos fueron explorados en CRIADO BOADO 1989a y CRIADO BOADO y FabRE. GAS VALCARCE 1989. 
Ahora bien, el estudio del emplazamiento no se puede limitar a concretar esos elementos, pues por ahí se llegaría a conclusiones equívocas como las que representan la relación con la geología y con vías de comunicación. En cambio deberemos buscar el sentido que se oculta detrás de esas relaciones. En este caso hablaremos de "vinculación" que, por lo tanto, es una categoría racionalizadora y de análisis abstrato, mientras que "dispocisión" es una categoría de carácter empírico y se utiliza en sentido descritivo.

Nuestra "mirada" al emplazamiento tumular, en vez de fijarse en los elementos concretos y tangibles directa e inmediatamente dispuestos al lado de las mámoas (rocas y caminos), deberá mirar a través de ellos para reconocer "a qué se "vinculan"» esas mámoas. Para ello necesitamos, evidentemente, un principio que determine qué debemos observar y ordene, además, la observación. Ese punto de partida surge de la propia e inherente racionalidad del hecho monumental, pues desde Giedion (1981) reconocemos que el constreñimiento básico de la arquitectura monumental es ser reconocida, ser vista; de donde se deriva que el estudio de la visibilización del monumento es un rango más básico del estudio y comprensión de ese monumento.

\subsection{Visibilidad y ocultación: la intencionalidad del monumento}

Desde 1984 se viene ensayando en Galicia este procedimiento, consistente en el análisis en rofundidad de las "condiciones de visibilidad del $y$ desde el monumento" ${ }^{12}$. Una vez admitido que la construcción tumular está en función de una "ógica de la visibilidad", que los túmulos han sido hechos para ser vistos, se deberá buscar sobre el terreno, y objetivar en base a análisis detenidos de carácter cartográfico y gráfico, desde dónde se ven los túmulos, planteándose a continuación preguntas del orden de: qué destaca de ellos, cómo se realzan, desde dónde se ven mejor, desde dónde se dejan ver, qué otras cosas se ven sobre, al lado y mejor que ellos. La sistematización de estas cuestiones ofrecerá una serie de recurrencias y rasgos íntimamente "vinculados" a los túmulos que podremos definir como los "factores uniformes" que operaron en el emplazamiento de esas construcciones. La descripción de esos factores se planteará en el próximo apartado. Pero antes nos detendremos algo más en el tema de la "visibilidad tumular».

\footnotetext{
12 Ver nota 2.
} 
Monumentos, nudos en el pañuelo. Megalitos, nudos en el espacio: Análisis...

Algunos casos en los que megalitos y túmulos parecieran estar ocultos podrían indicar que la "lógica de la visibilidad" no funciona de forma generalizada. Además de ello, la validez global de los presupuestos sobre la arquitectura megalitica en los que se basa dicha "lógica» tampoco está libre de discusión. Antes estas observaciones se puede responder de dos formas: desde un planto práctico a la primera y desde un plano teórico a la segunda.

Respecto a esta última destacaremos que el fenómeno tumular, en la medida en que es un fenómeno espacial, monumental y arquitectónico, está esencialmente vinculado con la visibilidad; en este sentido se debe reconocer que la finalidad fundamental del megalitismo y de las construcciones tumulares es "visibilizar la muerte" en sentido espacial y temporal. Es más, está bastante claro que aquel conjunto de la cultura material de una sociedad a la que llamamos "monumento" es en realidad cualquier construcción artificial concebida para mostrarse espacialmente y mantener esta función a lo largo del tiempo ${ }^{13}$.

Por otra parte, y desde un punto de vista práctico, debemos saber que las "condiciones de visibilidad" pueden ser muchas y muy variadas. Así resultará que ciertas mámoas que podrían parecer ocultas en realidad presentan unas circunstancias de visibilización específicas y originales. En primer lugar se debe diferenciar entre la concreción visual del elemento visibilizado y la abjetivación específica de esa visión que permite dotarla de un área propia, de una dirección e, incluso, de un posible sentido. En el primer caso optamos por utilizar el término "percepción", refiriéndonos con ello a la distinción dentro de un espacio considerado de un elemento determinado, en consecuencia, sería posible diferenciar entre la visibilización del túmulo en particular y la visibilización del grupo o necrópolis al que pertenece ese túmulo y/o de la unidad topográfica sobre la que se encuentra. A lo primero lo llamaremos percepción tumular y a lo segundo percepción zonal. Por motivos que se alegarán en el apartado 3.2., puede ser muy importante que la zona que ocupa una mámoa se diferencie e individualize con claridad dentro del conjunto del paisaje.

En cambio, reservaremos el concepto “visibilidad» para referirnos a la formalización de esa percepción en el segundo de los sentidos anotados en el párrafo anterior. Así, y en orden jerárquico, podríamos categorizar cuatro situaciones distintas. Denominaremos visibilidad "circular" al hecho

3 Esta definición parte, por un lado, de la crítica reciente en arqueología sobre el concepto de "cultura material" (HODDER 1989. SHANKS y TILLEY 1989) y, por otro, de una lectura arqueológica de las consideraciones sobre el monumento aportadas en RIEGL 1987. 
de que el ejemplo en cuestión se perciba nítidamente desde cualquier punto de su entorno. En cambio, visibilidad «sectorial» será cuando sólo se distinga desde un determinado sector que compone un abanico más - menos abierto. Por su parte, visibilidad «lineal» es un término que utilizaremos para referirnos a aquellos casos que sólo se individualizan adoptando una línea concreta de aproximación al monumento o figura topográfica. Por último, llamaremos visibilidad "puntual» a los casos que no se perciban en ningún momento con nitidez, salvo cuando se está al lado de ellos. (Toda la argumentación anterior se representa gráficamente en la figura 1).

\section{DESCRIPCIÓN: LOS FACTORES DEL EMPLAZAMIENTO TUMULAR}

Desde nuestro punto de vista, las circunstancias a las que se vinculan las mámoas se pueden agrupar en cuatro conjuntos fundamentales, cada uno de los cuales constituye un ámbito específico de análisis del emplazamiento y aparece como un factor de emplazamiento bien definido que, conjugado con los demás, genera esa realidad empírica que configura el mapa de situación megalítica.

Estos cuatro grupos los hemos denominado "geografía de la movilidad", "signos naturales", "referencias sociales» y "signos culturales». A través de ellos, y como se verá, intentamos ordenar el efecto ejercido por una serie de circunstancias de vinculación tumular caracterizadas respectivamente, en un sentido entre descriptivo y metafórico, por encontrarse "inscritas en" la naturaleza, "escritas por» la naturaleza, «inscritas sobre» la naturaleza, "escritas a pesar» de la naturaleza. A cada una de ellas dedicaremos un subapartado de este apartado.

De acuerdo con las observaciones recogidas en 2.1., no situamos a la "geología" entre los factores que regulan el emplazamiento tumular; creemos que la geología sólo es condicionante a un nivel empírico inmediato, pero que en realidad esa relación entre mámoas y configuración geológica refleja el efecto y actuación de otros elementos distintos. Insistiremos en que un trabajo que pretenda profundizar en el estudio del emplazamiento debería prescindir totalmente del factor geológico por cuanto las consideraciones relacionadas con él distraen e introducen una fuente adicional de «ruido" que no permite preveer la aparición de otros factores realmente más importantes. Tal actitud es asumida, al menos, por asepsia metodológica. 
Monumentos, nudos en el pañuelo. Megalitos, nudos en el espacio: Análisis...

Asi, por ejemplo, existen datos suficientes que muestran una disposición regular sobre sedimentos cuaternarios: graveras, terrazas, etc... ${ }^{14}$. Dado que no existen monumentos megalíticos construidos con losas de cantos rodados, resulta evidente que esa disposición no está en función de ningún determinismo de tipo geológico. En cambio, si se observan con detalle las condiciones topográficas y de visibilidad, se percibe que la vinculación de las mámoas a ese tipo de terrenos está en función de que esas zonas se corresponden con pequeñas "chairas» prominentes que permiten destacar el componente visual.

\subsection{La geografía de la movilidad: el tránsito}

Desde las tempranas observaciones por parte de Maciñeira (1947) en la Serra da Faladoira ( $N$ de la provincia de $A$ Coruña), se ha señalado la concordancia entre localización de los túmulos y el trazado de caminos viejos o reales. Aunque esta relación no fue destacada suficientemente por los arqueólogos gallegos clásicos ${ }^{15}$, sin duda desprovistos de la gran sensibilidad que Maciñeira había mostrado hacia la comprensión unitaria del paisaje ${ }^{16}$, esos mismos y otros autores reunieron diferentes datos en ese sentido ${ }^{17}$. En un trabajo previo se recogieron los ejemplos conocidos de esa relación (BELLO DIÉGUEZ et all. 1982). Este hecho no es exclusivo del megalitismo gallego, pues se ha señalado en otras zonas: Francia (Germont 1980), Portugal...

Estas coincidencias son suficientemente llamativas como para suponer que son indicativas de algo. Ahora bien, más allá de su mera constatación,

14 La mámoa de Monte Campelos (Begonte, Lugo) sería un ejemplo (Rodríguez CASAL 1983); otros serían el grupo de Reborica (Aranga, Coruña - VaQuero 1989a), el grupo del cerrado de Seara (As Pontes, Coruña, MaciñEIRA 1943), o el grupo de Monte do Cruceiro (Frades, Coruña).

15 Véase la "nota cautelar" de F. López CueviLlas en 1979: 56.

16 Uno de los trabajos pendientes de la historiografía arqueológica gallega lo constituye la obra de D. FEDERICO MACINEIRA y PARDO DE LAMA. Este precursor representa el tipo de propietario ilustrado, afirmado en sus posesiones rurales y comprometido con la renovación de las estructuras y técnicas agrarias, que en Inglaterra, desde el siglo xvill, dio pie al surgimiento de la primera arqueología como una práctica que extendía y completaba la identificación y preocupación de aquella aristocracia y burguesía liberal con el espacio que poseian (aunque no defiende esta tésis, se puede ver sobre este fenómeno LEVINE 1986). En Asturias la figura de $M$. SAENZ DE SAUTUOLA, en su doble proyección de fundador de la arqueología cántabra e introductor del eucalipto en Asturias (ver MADARIAGA et al. 1976: 15), representa ese mismo tipo de origen de la Arqueologia.

17 Véase por ejemplo: Amor Meillán 1918: 34, Barros Silvelo 1875: 77, Castillo López 1927, Diaz Sanjurjo 1902 y 1903, López Cuevillas 1925 


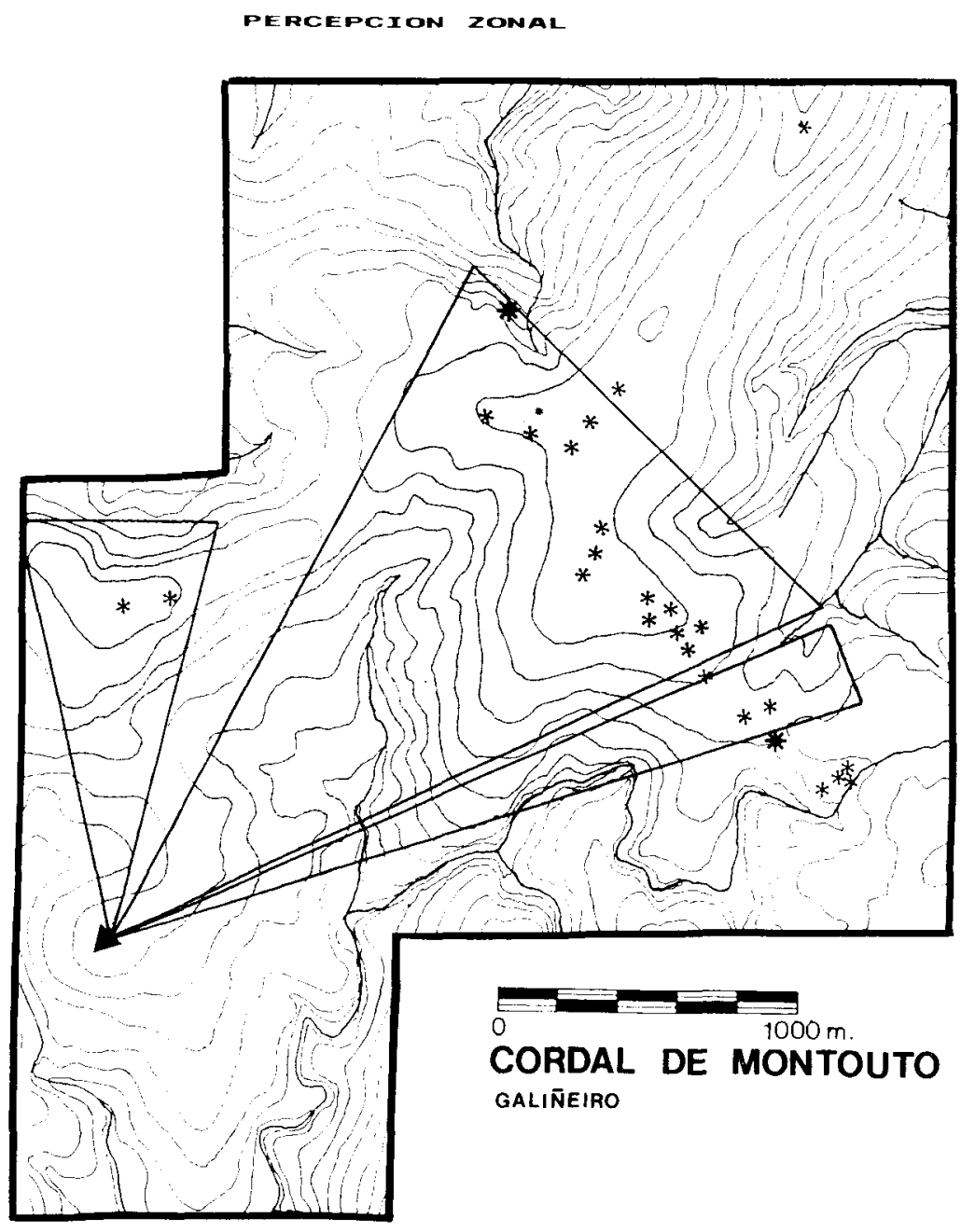

Fig. 1 
Monumentos, nudos en el pañuelo. Megalitos, nudos en el espacio: Análisis...

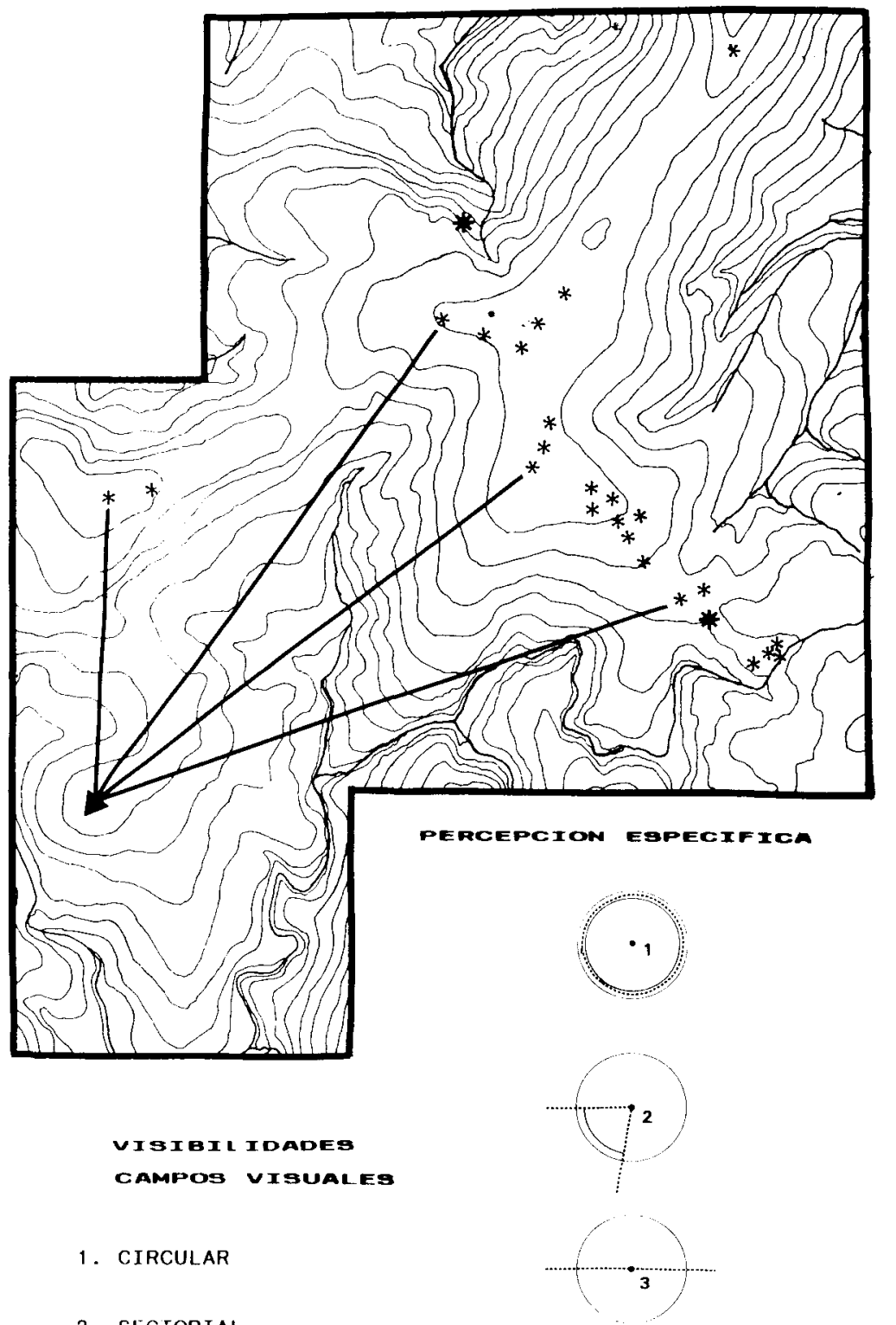

3. I. INEAL

4. PINNTUAL

Fig. 1. 
ningún trabajo anterior fue capaz de interpretar la relación entre túmulos y caminos. Por nuestra parte, creemos que la clave para entender esta vinculación consistirá en olvidarse un poco de la tiranía con la que se establece esta observación, dejar un poco de lado la omni-presencia del "Camiño» y fijarse en cambio un poco más en el «tránsito».

En efecto, en un trabajo intensivo de prospección megalítica de 94 túmulos realizado en los ayuntamientos de Aranga y Monfero (Coruña) y Guitiriz (Lugo) entre 1984 y 1988, se pudo observar la regular "asociación entre lugares de paso y disposición de túmulos" (VAQuero LAstres 1989b). Esta evidencia concluyó en la constatación de que la situación concreta de un elevado número de mámoas $y$, sobre todo, de las agrupaciones importantes de túmulos, estaba siempre en relación con lugares de tránsito utilizados indistintamente por los animales, los hombres y por las propias referencias míticas para comunicar dos áreas geográficas diferentes: tal fue el caso verificado en los grupos o necrópolis tumulares de A Reborica (VAQUero LAStRES 1989a) y Galiñeiro (VAQUERo Lastres 1990b).

De este modo, propondremos que uno de los factores que influyen en la localización de los monumentos tumulares es la "vinculación de los mismos a vías de tránsito", a zonas privilegiadas de comunicación. Esta asociación es, en el momento actual de nuestra investigación, el elemento más significativo del emplazamiento tumular no sólo porque es la circunstancia más fácil de observar y verificar empíricamente, sino sobre todo porque su efecto creemos que se deja sentir en todos los casos que se puedan considerar, dando lugar a una amplia variedad de tipos de emplazamiento que, en su conjunto, dependen de ese factor uniforme.

Esa variedad, desde nuestro punto de vista, está en función de formas específicas de tránsito, cada una de las cuales refleja una distinta «clave de desplazamiento" a través del terreno que se considera ${ }^{18}$. Precisamente, el análisis del emplazamiento desde esta perspectiva y el descubrimiento de la clave de desplazamiento significativa en cada caso, es lo que permite entender ciertas localizaciones tumulares que, a primera vista, parecerían aberrantes.

La definición teórica de los movimientos posibles dentro de un terreno determinado ha permitido delimitar una serie de líneas o claves de tránsito. Cada una de ellas se concreta en una forma fisiográfica específica que posibilita el desplazamiento a través de ella: "corda», “dorsal», "cruz", “Collado», "porto-vao» ${ }^{19}$. La “corda» recoge el tránsito en las zonas altas,

\footnotetext{
19 Véase este concepto en Vaquero Lastres 1990b.

19 Véase para toda esta descripción VAQUERO LASTRES 1990b y en lo que se refiere concretamente a los "portos" VAOUERO LASTRES 1990c.
} 
Monumentos, nudos en el pañuelo. Megalitos, nudos en el espacio: Análisis...

el desplazamiento a lo largo de una línea o divisoria de sierra. Este tipo de zona, al ser bastante llana y no presentar problemas de encharcamiento o de paso de corrientes de agua, resulta ideal para ser utilizada como vía de comunicación. Por su parte, la "dorsal de estribación» une las tierras altas con las bajas, y se utiliza generalmente para remontar el terreno desde una zona baja hacia zonas más elevadas. A ambas situaciones suelen vincularse una gran parte de los monumentos tumulares gallegos. En estos casos la visibilidad es de tipo específico general o sectorial fundamentalmente. Con todo, es frecuente encontrar un eje priorizado de visibilidad que coincide con la orientación del desplazamiento que se sigue.

La “cruz» es un concepto que en gallego y en Galicia se refiere al cruce de caminos de cierta entidad, y que puede coincidir, además, con un cruce fisográfico o topográfico. En un sentido teórico y referido a las dos claves anteriores, es la figura que resulta de la intersección de dos "cordas" o bien de una de éstas con sus "dorsales» más importantes; supone, en definitiva, el cruce de dos líneas de tránsito de cierta entidad. En un sentido metafórico es el cruce ante el cual todas las alternativas de desplazamiento que se puedan seguir poseen el mismo rango de prioridad. Este tipo de zonas poseen una relevancia especial. Generalmente se han realzado con la construcción de un "cruceiro" 0 , incluso, de una ermita y, además, poseen una destacada importancia dentro del folklore. La aparición de túmulos y necrópolis importantes en esta situación significa una vinculación señera con esa clave de tránsito. La visibilidad de los monumentos involucrados es mayoritariamente del tipo específico general.

El "collado" coincide, sobre todo, con una zona deprimida a través de la cual se atraviesa transversalmente una zona más elevada, normalmente divisoria; soluciona el obstáculo que representa una cuerda situada entre dos zonas bajas para aquél que desee pasar de un lado a otro. Las mámoas que se emplazan vinculadas a esta clave de tránsito presentan condiciones de visibilidad tumular lineal y sectorial: de un modo lógico y coherente con el sentido del tránsito, la mejor y, a menudo, única forma de ver el túmulo es aproximarse a él siguiendo la orientación del tránsito.

Finalmente definimos el "porto/vao" como la clave de tránsito que soluciona el obstáculo más importante para el movimiento a través de topografias deprimidas: las corrientes de agua. Ambas denominaciones significan en gallego el punto por el que los animales y vehículos de tracción animal pueden sortear una determinada corriente de agua sin necesidad de obra artificial alguna, si bien en el caso del "porto" se suelen disponer unas pequeñas losas de piedra que alisan la superficie de paso (BÁs 


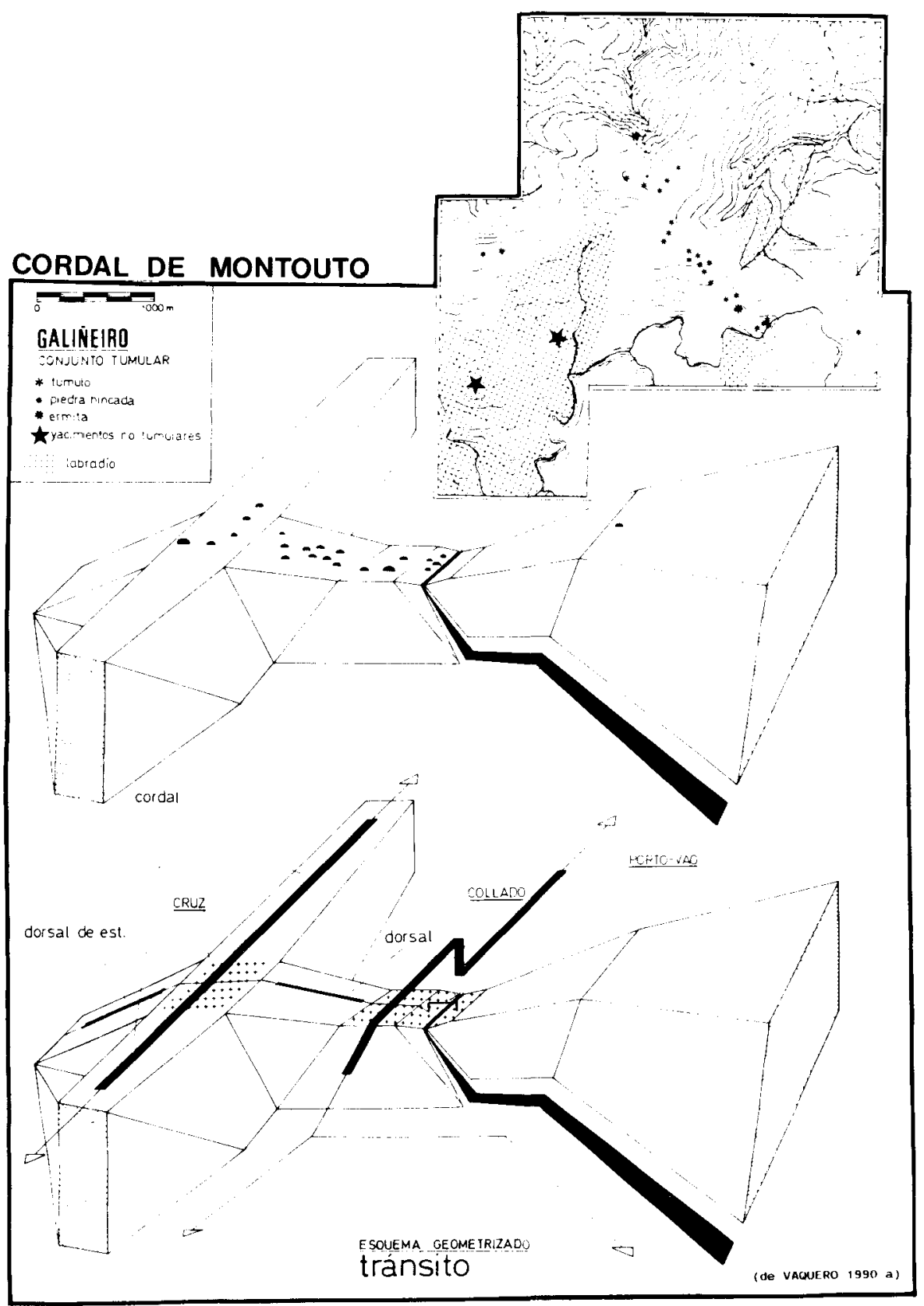

Fig. 2. 
Monumentos, nudos en el pañuelo. Megalitos, nudos en el espacio: Análisis...

1985). La definición de este tipo de desplazamiento es de excepcional importancia por cuanto permite entender la presencia de mámoas en zonas deprimidas y ocultas o semiocultas que, en principio, parecerían ocupar posiciones aberrantes y que irian directamente en contra de los principios que se derivan de la "lógica de la visibilidad». Los túmulos vinculados a "portos" presentan siempre una visibilidad tumular lineal o en abanico muy reducido. Desde nuestro punto de vista, los monumentos construidos en esta situación y en la anterior fueron levantados para presidir una zona de tránsito de especial relevancia y por esa razón únicamente se individualizan visualmente como tales monumentos cuando uno asume la dirección del tránsito que se realiza(ba) a través de la zona en la que se emplazaron. (Estas situaciones se expresan de forma gráfica en la figura 3.)

Si a partir de las bases anteriores retomamos la relación entre mámoas y "camiños reales", pero lo hacemos abandonando una escala exhaustiva y general y adoptando en cambio una escala de observación de detalle, se comprueba que la situación de cada grupo de mámoas o, incluso, de ciertas mámoas individuales, se vincula, además de a la línea longitudinal de movimiento que representa el "camiño real", a zonas de paso y de tránsito transversales a la anterior.

Este es el caso, por ejemplo, de la Sierra de O Bocelo (Coruña), en la que 29 de las 32 catalogadas se sitúan alineadas con el "Camiño Real» que la cruza en sentido ENE-WSW, discurriendo a lo largo de la divisoria principal de la sierra. Sin embargo, en un sentido más concreto la totalidad de los túmulos identificados, e incluso aquellos que se encuentran situados fuera del camino, se localizan en zonas que se corresponden siempre con "cruces", "collados" o "rellanos" de la sierra y que constituyen las zonas de paso, ya no a lo largo de la sierra, sino a través de ésta, entre las cuencas del Ulla y del Tambre divididas por la citada sierra (CRIADO, Bonilla et all. 1990a) ${ }^{20}$. En la mayor parte de los casos que podemos reconocer, esa función de paso posibilitada por la topografía no es un mero constructo hipotético, sino que coincide con la presencia real de caminos importantes que han sido utilizados durante largo tiempo, de acuerdo con las informaciones etnográficas y que, en algunos casos, concuerdan con el trazado de las carreteras construidas en la actualidad para unir los dos valles anteriores entre sí.

20 En un caso concreto (PA 13) el collado en cuyo eje se sitúa una mámoa imponente, es también el eje por el que atraviesa un camino de trazado antiguo que de un lado empieza en un castro (castro de A Orela) y de otro está presidido por una estructura defensiva medieval (Torre dos Mouros). 


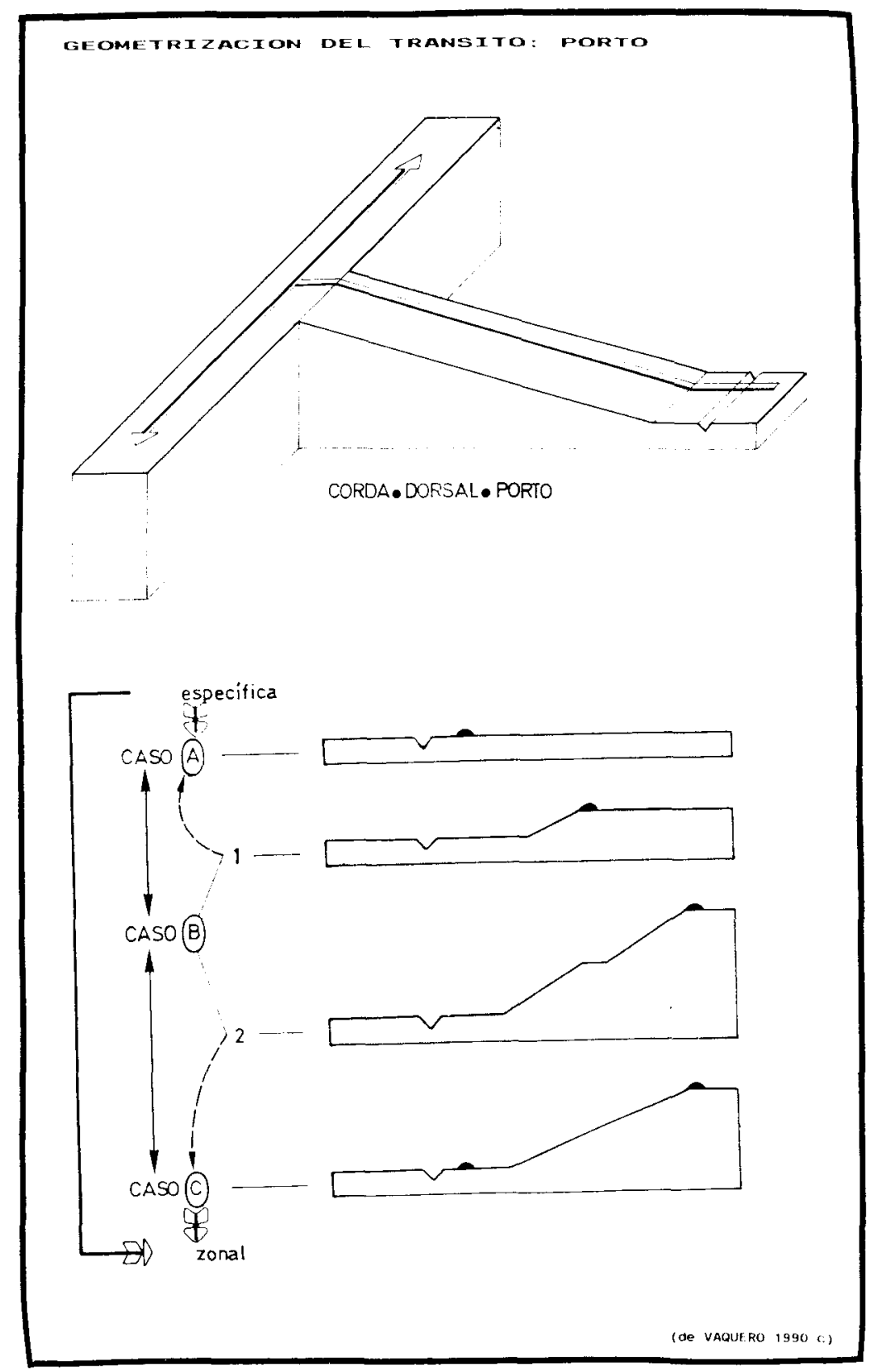

Fig. 3. 
Monumentos, nudos en el pañuelo. Megalitos, nudos en el espacio: Análisis...

Por otra parte, para reconocer las auténticas funciones de paso de esos collados "megalitizados", se ensayó una estrategia de trabajo consistente en seguir el tránsito de los caballos que viven en régimen de libertad en el monte (INFANTE et all. 1990). Aunque este intento no ha constituido más que una aproximación, el resultado no pudo ser más prometedor, ya que se verificó que las zonas de paso utilizadas sistemáticamente por el ganado coincidian con la localización de los túmulos.

A través de todas estas consideraciones lo que se percibe es la vinculación del mundo tumular con un fenómeno muy vasto y sin duda complejo que podríamos denominar «geografía de la movilidad». En efecto, yendo más allá de las propuestas simplistas que podrían conducir a relacionar directamente el emplazamiento tumular con vías naturales de comunicación, debemos entender que si bien por debajo de cualquier vía de tránsito aparece una determinada topografía que posibilita la realización de este tránsito por encima de esa forma de la geografía física el hecho mismo del tránsito establece una geografía móvil (cuando el tránsito pertenece al orden de la naturaleza) y/o una escala humana (cuando pertenece al orden de la cultura) que provee de una significación especial al paisaje. Este paso, de una mera determinación ecológica o comportamiento etológico a una concepción cultural, se apoya en el reconocimiento de que cualquier comportamiento social es ante todo costumbre cultural $^{21}$.

Sin duda el movimiento a través del espacio natural está en parte ya «inscrito en la configuración natural» de ese espacio. Pero además de ello, el movimiento es sobre todo una "experiencia» del espacio natural $y$, en este sentido, su concretización con elementos artificiales es un modo de construir un espacio cultural utilizando esa experiencia previa. Sobre este umbral de significación creemos que se resuelve en gran medida el emplazamiento tumular que a través de estas consideraciones se nos aparece vinculado a una geografía de la movilidad y deja de estar constreñido a un espacio inmóvil. Lo que esto pueda representar lo exploraremos más adelante, en el apartado 3.4 .

\subsection{Los signos naturales: rocas y cuarzos}

Los elementos que se considerarán en este apartado derivan, al igual que los anteriores, del orden natural. Si bien es cierto que, a diferencia

21 Como es bien sabido, en contra de la tendencia de la Nueva Arqueologia que ha insistido en reducir lo social al mero comportamiento humano empíricamente observable, la crítica reciente de esa estrategia ha insistido en este otro sentido; un ejemplo concreto aplicado a esta noción se puede encontrar en el breve comentario de BARAET 1983. 
de ellos, no representan un factor de emplazamiento tan constante como el generado por la "geografía del movimiento", propondremos que otro factor de emplazamiento uniforme es la vinculación de muchos túmulos con ciertos elementos naturales que habrían poseído una significación especial 0 , en torno a los cuales, todavía hoy se percibe una cierta morfología específica.

En este sentido, y una vez que rechazando el determinismo geológico rechazamos asimismo que posea la más mínima importancia la vinculación a la materia prima y a las canteras, la disposición de algunas mámoas al lado de afloraciones de "rocas" y de "penedos" creemos que podría en. tenderse en un sentido simbólico.

Evidentemente se podría argüir que esos casos muestran una cierta vinculación a las canteras. Pero lo que nos lleva a pensar que esa asociación debe ser entendida de otra forma es el hecho de que, en los casos que conocemos, las afloraciones más próximas no fueron utilizadas nunca como los puntos de obtención de las losas del megalito $\mathrm{y}$, en algunas ocasiones, el material de aquellas ni tan siquiera coincide con el utilizado para levantar el monumento. En cambio, las rocas anejas suelen presentar unas características de visibilidad que contribuyen a destacarlas en sentido especial: por su situación, volumen y/o color esas rocas se divisan desde distancias considerables. Parecería entonces que la disposición de los túmulos al lado de las mismas es una forma de realzar la visibilidad de éstos y de incrementar su monumentalidad a través de una cierta monumentalidad natural.

Evidentemente se dirá que esta apreciación es demasiado subjetiva e incontrastable. Tal vez sea cierto. Pero no es menos cierto que podemos añadir algunas otras evidencias que fundamentan la observación anterior y que, al mismo tiempo, permiten completar la definición de este factor de emplazamiento.

En este sentido, la primera que nos ofrece el registro gallego son los ejemplos de monumentos megalíticos en los que la cámara ha sido construida utilizando y aprovechando una afloración de roca del sustrato. Al. gunos ejemplos aportados por Maciñeira y pertenecientes a Serra Faladoira permiten documentar que, si bien este fenómeno no es general, tampoco es excepcional o accidental (Monte do Grau, MACINEEIRA 1947). Se podría entender que estos casos de "reutilización de rocas naturales" como parte de la estructura megalítica muestran una voluntad de minimización de esfuerzos en la construcción de la cámara. Reaparecería así el determinismo geológico. Sin embargo, la abundancia de materia prima en las inmediaciones, la facilidad de extracción de losas y el pequeño tamaño de las lajas con las que se construyó la cámara, minimizan al 
máximo el volumen de esfuerzo que habría requerido el desprendimiento y transporte de las losas "sustituidas». Ante ello parece que detrás del aprovechamiento de esas afloraciones aflora una voluntad de orden especifico y que no es fundamentalmente de orden economo-energicista.

En los casos que se han descrito, la roca reutilizada es muchas veces "cuarzo blanco", mientras que el material utilizado en el resto de las losas de la cámara puede ser de otro tipo, principalmente pizarra. Ahora bien, si tenemos en cuenta que el cuarzo blanco aparece en algunos casos utilizado sistemáticamente y casi de forma exclusiva en la construcción de las "corazas tumulares", si a ello le anadimos que en ocasiones los bloques de cuarzo de la coraza fueron obtenidos en un punto distinto a aquél en el que se levantó el túmulo y, finalmente, si tenemos en cuenta que las corazas de cuarzo blanco manifiestan una calidad especial que permite diferenciarlas desde muy lejos (es el caso del túmulo 2 del Bocelo - Coruña- que responde además al significativo nombre de «Crollos Brancos»), podríamos suponer que el aprovechamiento del cuarzo en ambos casos (reutilización en la cámara y construcción de la coraza) no es un fenómeno accidental sino que exhibe una significación específica de este material.

Tamaña sugerencia podría, nuevamente, ser tildada de excesivamente subjetiva. Pero de nuevo acude en apoyo de esta posibilidad una fuente de datos complementaria. Conocida es la frecuente aparición de «prismas de cuarzo" formando parte de los ajuares megalíticos. Fábregas (1990) ha reunido toda la evidencia en este sentido y ha documentado que su presencia como parte de ajuares es bastante sistemática. Al mismo tiempo se ha señalado la probable significación simbólica de dichos primas que, entre otras cosas, aparecen en ciertas culturas como representaciones metafóricas del sol y la luz divina. No vamos a extendernos en esta línea, que supondría usar y abusar de la información disponible en sentido transcultural. En cambio creemos que los datos anteriores evidencian suficientemente una inconcreta pero evidente adjetivación especial del material de "cuarzo".

Existen otros dos elementos que cobran asimismo sentido ante la circunstancia anterior. Algunas excavaciones realizadas en túmulos han verificado que la construcción de los mismos se habia realizado sobre «vetas de cuarzo blanco" de unos pocos metros de anchura, diluidas en el seno de una mancha geológica de un material distinto y que no se diferenciaban superficialmente ${ }^{22}$. Teniendo en cuenta que el desplazamiento de unos

22 Dos ejemplos: túmulo n. ${ }^{\circ} 6$ de A Cruz do Bocelo, Coruña, (Criado, Bonilla et al. 1990b) y mámoa de "Chousa Nova», Pontevedra, Silleda, excavada en 1985 por J.M. Bello Diéguez. 
pocos metros del punto de levantamiento del monumento habría supuesto que éste en vez de encontrarse sobre el sustrato de cuarzo, se localizase sobre un material distinto, es posible que debamos considerar que en esos casos la asociación entre el elemento natural (el cuarzo) y la construcción artificial (el túmulo), en vez de responder al accidente o la casualidad, fue algo buscado explícitamente por el grupo constructor.

El segundo elemento se documentó por primera vez en la excavación del monumento de "Forno dos Mouros" (Bocelo, Coruña, CRIAdo y VA. QUERO 1990). En este caso el megalito estaba construido sobre sustrato granítico, material que se utilizó en todas las losas de la cámara. Sin embargo la excavación documentó que la mayor parte de los «calzos" de las losas de la cámara eran de cuarzo blanco. Dado que estos calzos, tanto por su pequeño tamaño, como por su escaso número y colocación, no desempeñaban función práctica alguna, no parece probable que el material de cuarzo fuese elegido por razones de orden funcional como pudiera ser su mayor resistencia. En cambio volvemos al punto en el que la utilización del cuarzo en la base del monumento y en el momento de inicio de su construcción pareciera llevarnos a un orden distinto.

Los datos anteriores parecen manifestar una misma regularidad, aún inconcreta pero evidente. Ante ella nos podríamos extender alegando todo tipo de consideraciones subjetivo-simbólicas sobre el significado del cuarzo. Pero, como se dijo antes, no es esa nuestra intención ni forma de proceder. Creemos en cambio que el conjunto de los datos recogidos sugieren que un factor posible de emplazamiento tumular haya sido la vinculación de los monumentos a circunstancias naturales señeras.

En este mismo sentido, la investigación reciente que se desarrolla dentro de la sierra de $O$ Bocelo permite verificar que las áreas de enterramiento megalítico coinciden con las áreas de caza de las comunidades paleolíticas y epipaleolíticas ${ }^{23}$ (véase en este sentido la Figura 4). No propondremos que esa relación sea consciente y que implique una continuidad en la utilización de esas áreas por parte del hombre, incluso si, como parece ocurrir, las áreas megalíticas dotadas de buena visibilidad zonal coinciden con zonas en las que se concentran los yacimientos paleo-epipaleolíticos. En cambio, si tenemos en cuenta que las sociedades pre-campesinas y que llamamos "primitivas" ${ }^{24}$ conciben un espacio imaginario que se apoya, no en la construcción de elementos artificiales, sino en la utilización de elementos naturales adjetivándolos de una forma es-

${ }^{23}$ Véase sobre estas últimas CEROUEIRO LANDÍN 1989 y 1990.

24 Sobre este concepto y temática ver CRIAdo 1989a y CRIAdo y PENEdo 1989. 
pecial ${ }^{25}$, no habría sido imposible que la construcción de la monumentalidad megalítica se hubiera basado también en el aprovechamiento de elementos naturales que, dotados de un prestigio simbólico dentro de una concepción previa y distinta del paisaje ${ }^{26}$, siguieron manteniendo este prestigio especial cuando se descompuso ese paisaje anterior, la cultura que lo pensó y la sociedad que lo generó.

El problema esencial de los datos y observaciones que aqui se han recogido no estriba en que sean subjetivos e incontrastables, sino en que su aparición no es predecible, constante o sistemática y, por lo tanto, su estudio debe ser hecho a posteriori y utilizando sólo los casos, tal vez excepcionales, en los que parece documentarse una asociación de este tipo entre túmulos y elementos naturales. A pesar del carácter episódico de esta circunstancia, creemos que se puede definir como un factor de emplazamiento uniforme la vinculación de los monumentos a cierto elenco de elementos naturales señeros. Esos elementos están "escritos por el propio orden de la naturaleza" $y$, en ese sentido ${ }^{27}$, son una especie de "signos naturales" en los que apoya la monumentalización megalítica.

\subsection{Las referencias sociales: espacio y hábitat}

El estudio exhaustivo de la distribución de túmulos de diferentes regiones gallegas desde el punto de vista del "asentamiento" y de su relación con el ambiente, permite extraer algunas observaciones que, aunque referidas a una escala y temática totalmente distinta a la que ahora nos ocupa, ofrecen datos importantes para definir el juego de factores que influyen en el emplazamiento tumular.

En diferentes comarcas prospectadas y estudiadas sistemáticamente en los últimos años se puede apreciar una configuración especial de la distribución general de mámoas que responde al esquema idealizado de una distribución bandeada. Con ello queremos decir que la presencia y abundancia de túmulos en una zona determinada, es replicada por su ausencia o rarificación en otras áreas concretas: en los mapas de distribución general zonas con mámoas se oponen a zonas sin ellas. En otros

25 No es éste lugar ni ocasión para extendernos en este sentido, pero sobre este tema, en parte, se pueden ver los primeros capítulos de LÉvI-Strauss 1964.

${ }^{26}$ A esta concepción la llamaríamos "salvaje»; ver nota 24.

27 Pero sólo en ese sentido, pues un signo, por el mero hecho de serlo, no es natural, sino cultural; en sentido estricto, siguiendo a Barthes (1980) diríamos que el aspecto natural es el referente de un signo cultural. 


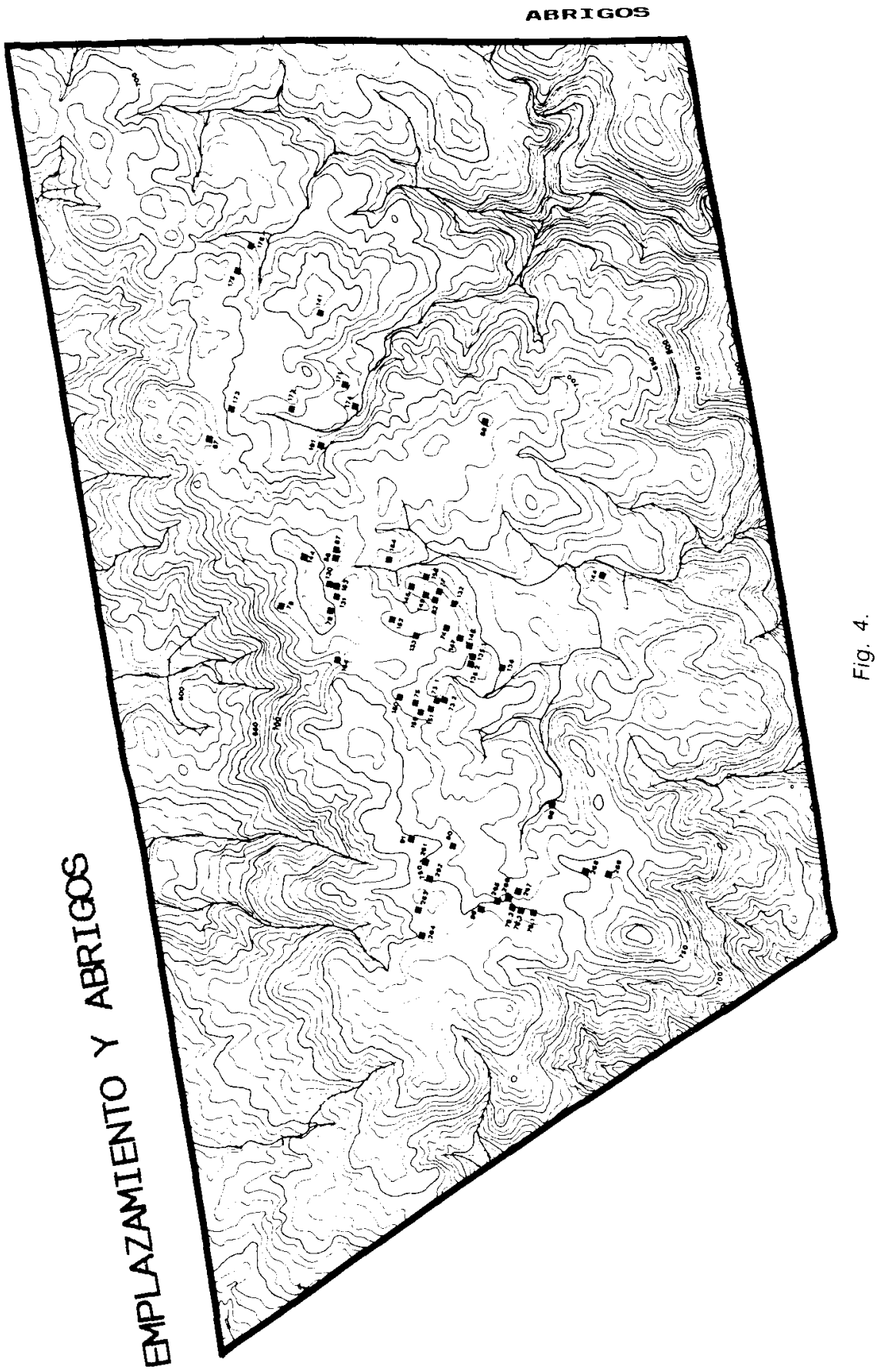


Monumentos, nudos en el pañuelo. Megalitos, nudos en el espacio: Análisis...

\section{BOCELO-FURELOS}

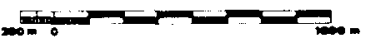

TUMULOS

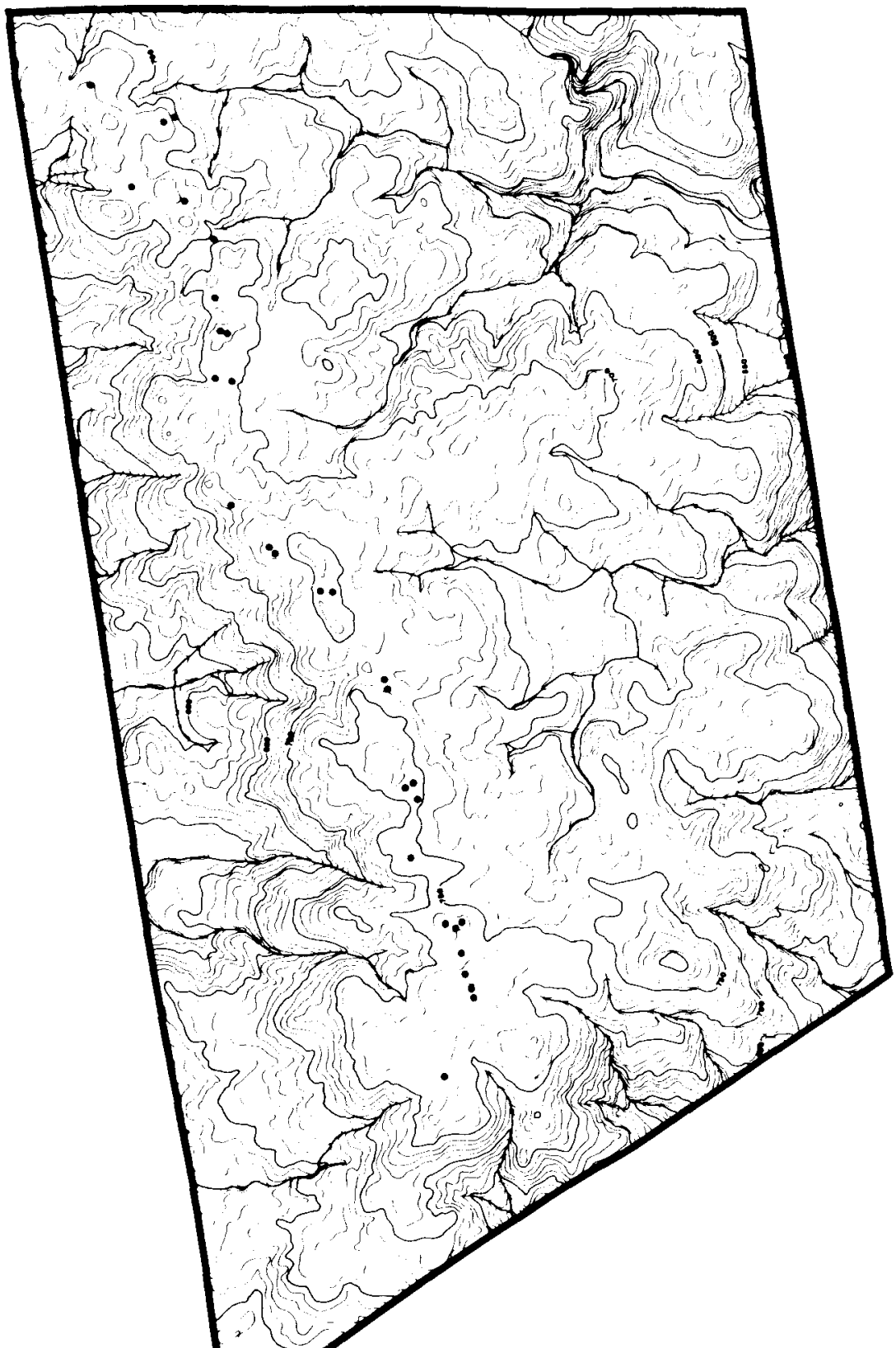

Fig. 4. 
trabajos se ha utilizado esta dualidad para, observándola desde una escala regional, caracterizar la ocupación megalítica del espacio y definir sus relaciones con circunstancias ambientales, tecnológicas y subsistenciales (CRIADO 1989b y 1988).

Ahora bien, este mismo hecho, considerado en esta ocasión desde una escala de mayor resolución, nos permite apreciar lo que creemos que es un "tercer conjunto de factores" determinantes del emplazamiento tumular. Para definir este nuevo factor de emplazamiento procede entender la distribución megalítica desde una adecuada conceptualización del paisaje rural sobre el que se asienta.

En Galicia el espacio rural tradicional ha estado, y todavía sigue estando, repartido entre tres tipos distintos de terreno. Por un lado tenemos los campos de trabajo intensivo y constantemente ocupados que constituyen el «labradío» o "agro»; en su conjunto ocupan una superficie bastante pequeña que no excede la tercera parte de la superficie total ${ }^{28}$. Por el otro lado se encuentran los terrenos de trabajo extensivo, utilizados tradicionalmente para pasto extensivo del ganado, para el cultivo de cereal con sistema de roza y, sobre todo, para la obtención del tojo que, unido a los excrementos de los animales, se utilizaba más tarde para abonar el primer tipo de terrenos y evitar el descenso de productividad de los mismos; este tipo de tierras se denominan en gallego "o monte" y ocupan la mayor parte de la superficie restante; a pesar de su apariencia inicial, estos terrenos no se pueden conceptualizar como «inculto». El «inculto» propiamente dicho ocupaba sectores muy reducidos y marginales dentro del espacio agrario; solía coincidir con zonas de relieve abrupto, divisorias - que presentaban problemas específicos para su aprovechamiento; en cualquier caso hacían de límite superior de una malla de ocupación del espacio dispersa y diseminada.

Si basándonos en esta caracterización del espacio agrario tradicional, observamos los mapas de distribución de mámoas de comarcas bien prospectadas, podremos definir mejor la "configuración bandeada" de esa distribución. De este modo se descubre el efecto de un tercer factor de emplazamiento tumular que no sería ni más ni menos importante que los dos tratados anteriormente, pero que plantea el interés de relacionar la dimensión simbólica de la monumentalidad megalítica con la dimensión social y material de ese fenómeno.

${ }_{28}$ Sólo en ciertas zonas gallegas, como las rias bajas, en las que se han practicado desde el siglo xvil formas de intensificación agraria basadas en el cultivo de ciertos productos especializados (maiz, vid), este tipo de terrenos ha aumentando hasta llegar a ser mayoritarios. 
En efecto, esa "distribución bandeada" del mapa de túmulos se corresponde en líneas generales con la propia configuración bandeada del espacio agrario generada por la sucesión y alternancia de los tres tipos de terrenos que se acaban de distinguir. Así, si por un lado resulta que el espacio agrario puede esquematizarse en base a la sucesión reiterativa de: labradio-inculto, por el otro encontraremos que la situación de las mámoas se concentra en general en el segundo tipo de terrenos y, más en concreto, en el umbral intermedio entre los primeros, de trabajo intensivo, y los segundos. Esta triple parcelación, por otra parte, se inscribe sobre un tipo de perfil topográfico que define un "paisaje cóncavo" y en el que se suceden una zona deprimida, una zona elevada intermedia y una zona de divisoria superior.

En este sentido, los túmulos se disponen preferentemente en la segunda región topográfica, orientados de tal modo que su visibilización es sobre todo muy buena desde las zonas bajas anejas, mientras que se confunden si se contemplan desde su espalda. En algunos casos se encuentran mámoas en el tercer tipo de zonas. Lo característico de estos casos, que resultan excepcionales (mientras las grandes densidades de túmulos ocupan el umbral topográfico anterior), es que los monumentos que ocupan esa posición son sin duda los que poseen un tipo de visibilidad tumular general de ámbito más amplio, lo que parece sugerir que fueron vinculados a esa localización por motivos especiales. (Véase este patrón distributivo esquematizado en la Figura 5).

En cualquier caso los ejemplos más frecuentes de distribución microcomarcal señalan una clara tríada en la que por un lado las tierras incultas e inhóspitas de relieve accidentado y de escaso suelo, se oponen a un grupo de tierras más bajas y asequibles en las que, por su parte, se oponen la zona en la que se sitúan las mámoas a las zonas más bajas inmediatas en las que se concentra el hábitat tradicional. Ejemplos de este tipo se han registrado en: valle interior de la cima de la sierra de Barbanza (Coruña, CRIADo et al 1986), parroquia de Cidadela en el valle del Cabalar (Sobrado, Coruña), valle del río Deo (Aranga y Curtis, Coruña), parroquia de Illán en el valle del Ladra (Begonte, Lugo); los datos de campo de todos estos casos están recogidos en las memorias depositadas por los autores de este trabajo en la Dirección Xeral de Cultura de la Xunta de Galicia. (En las Figuras 6 y 7 se ofrecen varios ejemplos de este fenómeno representados gráficamente).

Así pues, propondremos que esta "configuración bandeada" del espacio tumular, cuando se considera a escala de detalle y de forma exhaustiva, (a distancias entre 100 y 500 metros para entendernos), refleja el efecto de un tercer factor de emplazamiento constituido en este caso 
FELIPE CRIADO BOADO Y JACOBO VAQUERO LASTRES

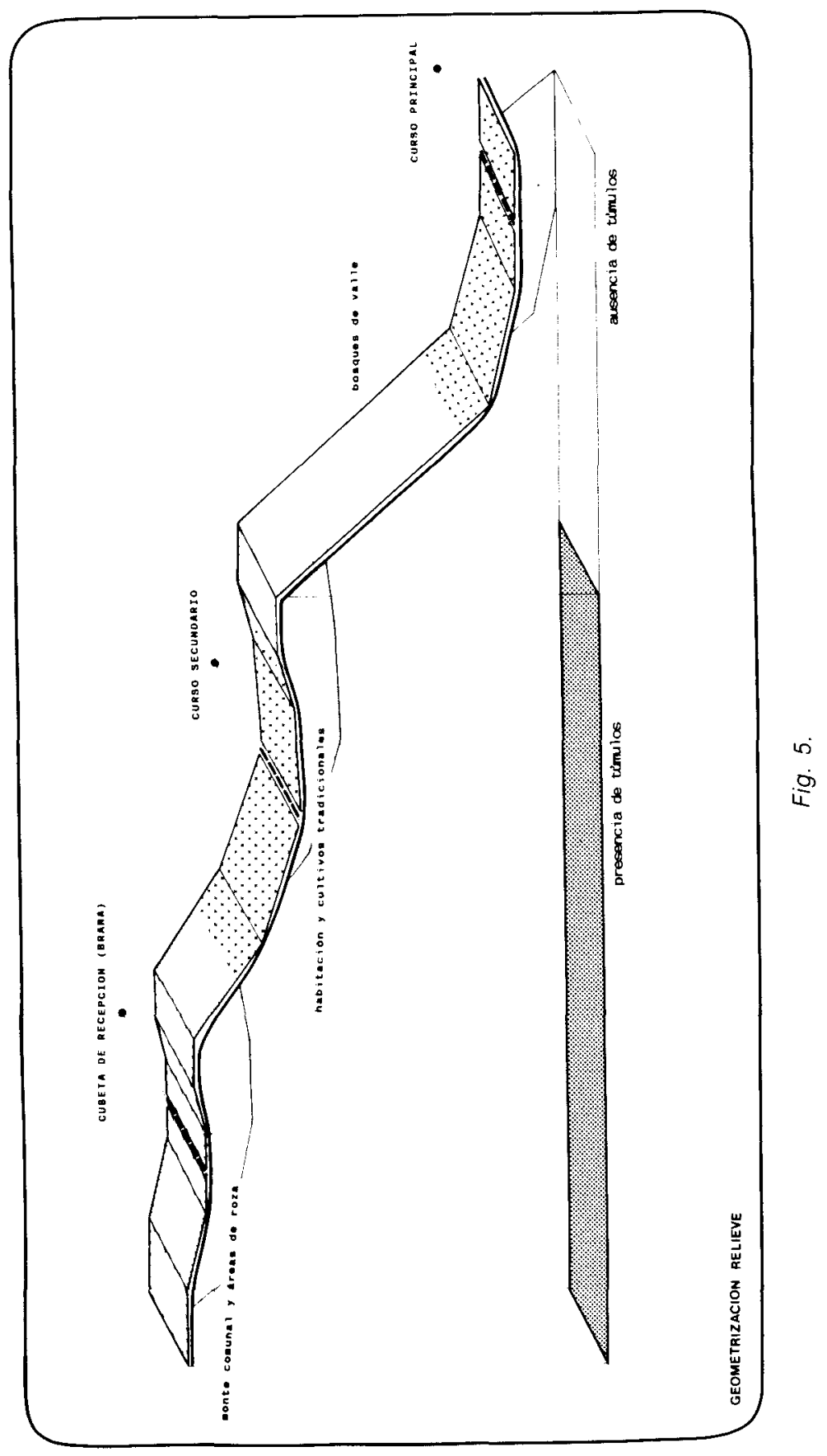


Monumentos, nudos en el pañuelo. Megalitos, nudos en el espacio: Análisis...

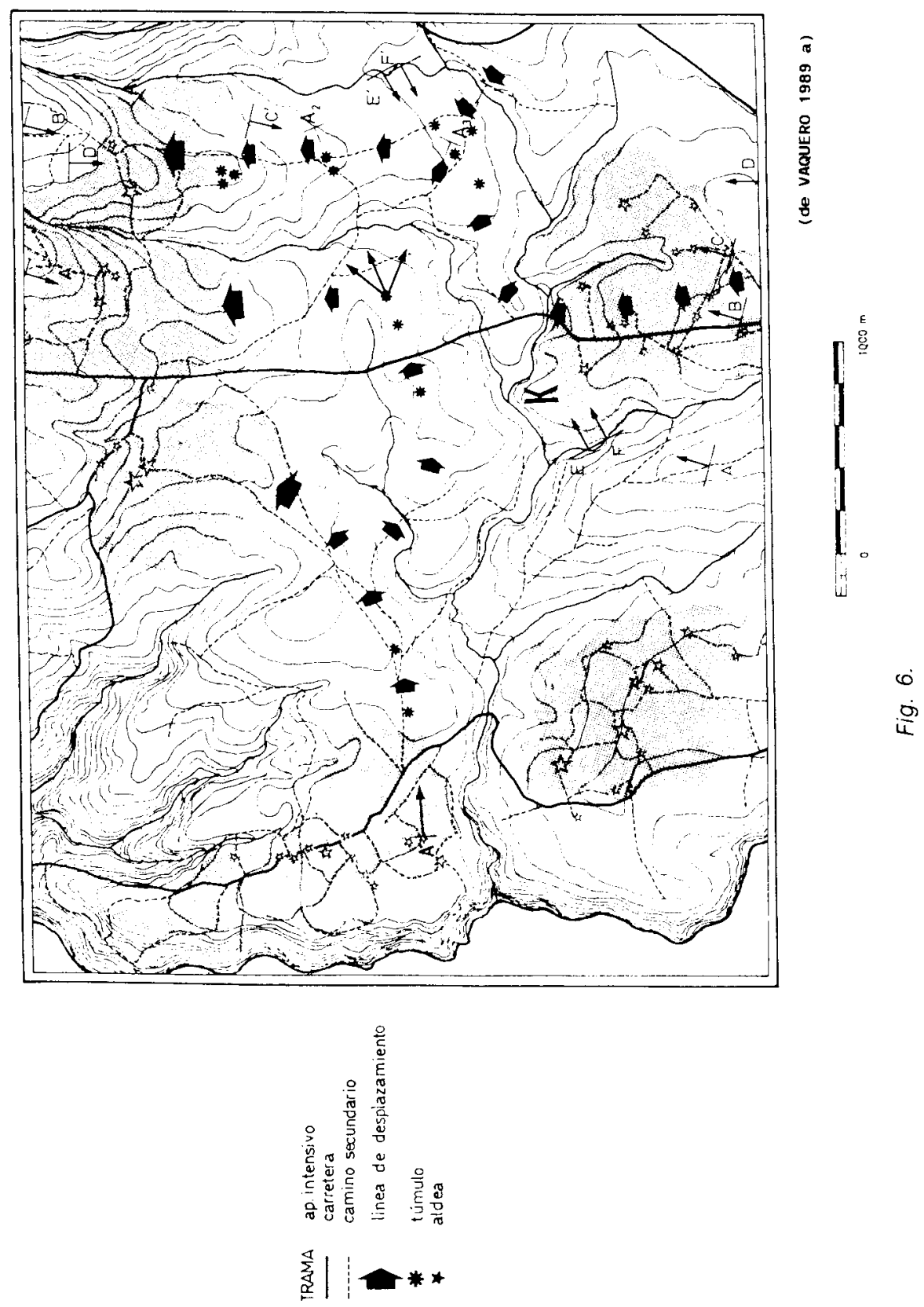



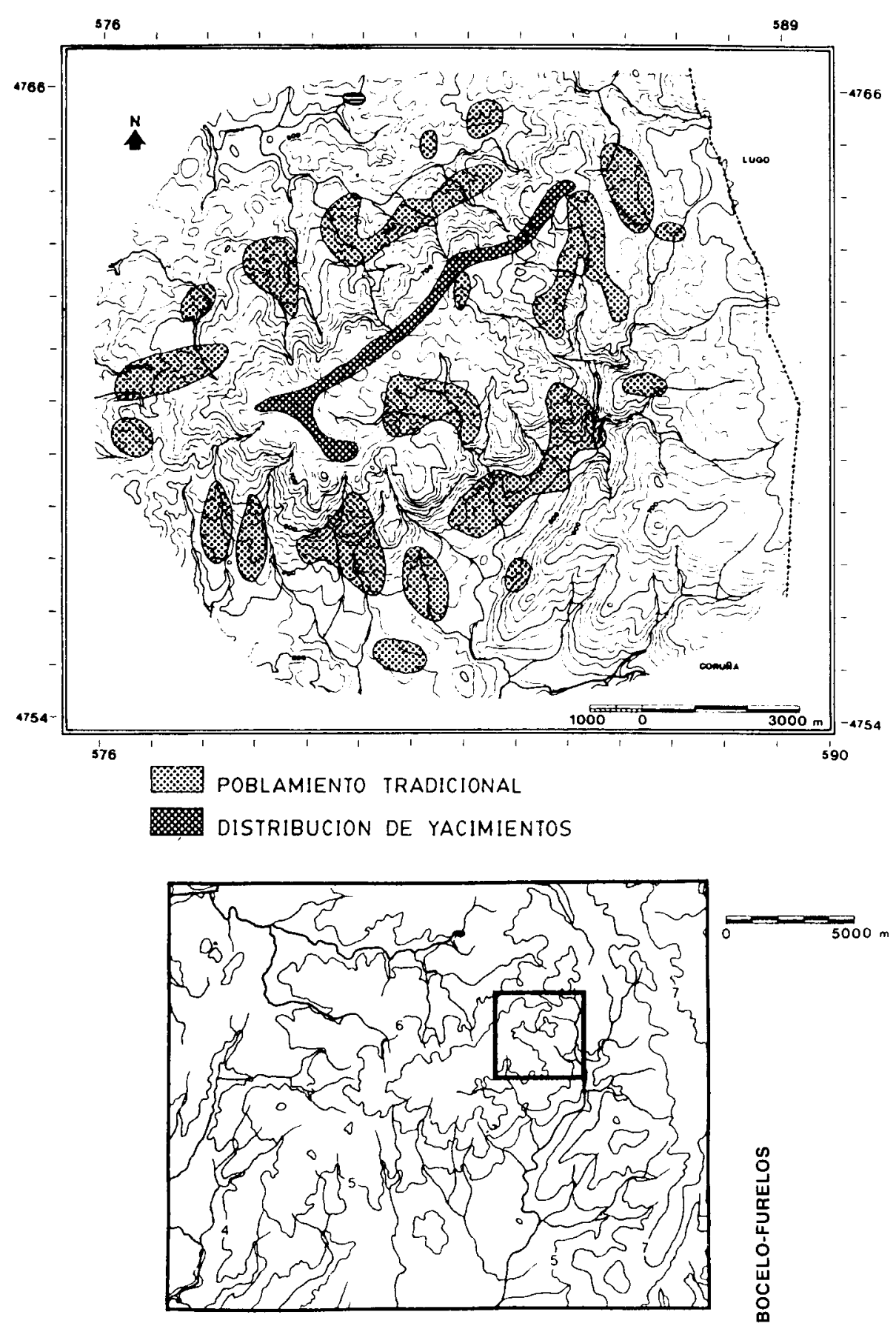

Fig. 6. 


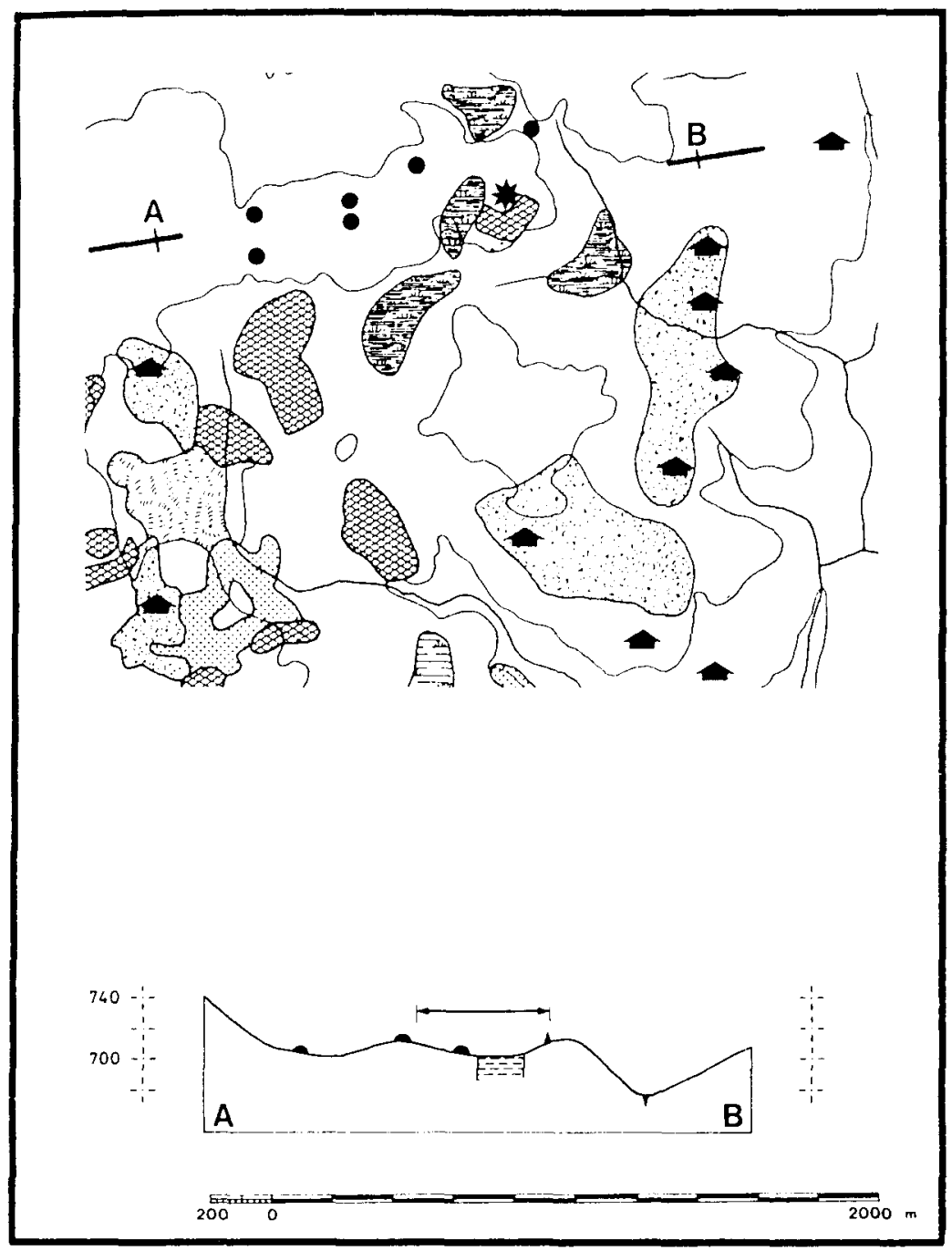

REI PINAR - EUCALIPTAL. B. ROBLEDAL RETAMAR

$\square$ TOJAL - BREZAL

- aldea actual

- tumulo
VEGETACION HIGROFILA IURFOFILA PRADOS

[a] CULTIVOS

[...]ROZAS

Fig. 6 . 
por la referencia del espacio social y cotidiano de las comunidades que habrían construido los monumentos. Creemos que la alternancia espacio abrigado::espacio tumular-funerario concreta el efecto de ese tipo de factores. En la medida en que esa parcelación del espacio está en un sentido no determinista prefigurada por la propia configuración del espacio ecológico, pero en función además de que sólo la acción social y el trabajo humano constituyen la fuerza que crea sobre ese medio un espacio social, diremos que este tercer tipo de elementos determinantes del emplazamiento tumular se encuentran «inscritos sobre la naturaleza". La actuación de este grupo de factores creemos que opera de un modo uniforme y que se presenta de forma bastante constante, si bien no en la totalidad de los ejemplos que se consideran.

Evidentemente hay muchas dificultades implicadas en esta propuesta. El primer grupo de problemas estriba en que estamos preestableciendo que las comunidades constructoras de los túmulos poseían un amplio grado de sedentarización y que situaban sus hábitats en relación inmediata con sus monumentos. La segunda dificultad viene dada por la ausencia de asentamientos megalíticos para contrastar esta hipótesis. Dado el carácter predictivo de estas propuestas, su certeza sólo podrá ser confirmada o rechazada en el futuro en función de la aparición o no de algún tipo de asentamiento megalítico en la posición que sería de esperar de acuerdo con ellas. En este sentido, además, comentaremos en el apartado final de este trabajo los primeros datos que tenemos sobre esta cuestión que, creemos, confirman el planteamiento asumido.

\subsection{Los signos culturales: movimiento y tradición}

Del mismo modo que las observaciones previas se basan en la asunción de que las relaciones definidas no son consecuencia de la mera casualidad, existe otro nuevo elemento ( $y$ por el momento último) con el que también están estrechamente relacionadas las mámoas en múltiples ocasiones. Nos referimos a la disposición de una mámoa al lado de otras mámoas construidas previamente. Nos referimos en definitiva a esa relación que ha llevado a hablar en muchos casos (y no sólo en Galicia) de la agrupación de túmulos formando "necrópolis".

Sin duda son muchos los problemas que encierra ese término y por ello, en aras de la rigurosidad, se ha preferido a veces dejarlo de lado y no utilizarlo. En un sentido estricto hablar de "necrópolis" implica reconocer una identidad de utilización y planteamiento que subyacería a todos los monumentos que configuran la "necrópolis", lo que conduce a suponer que todos ellos reunidos formaron un conjunto uniforme, creado por la 
Monumentos, nudos en el pañuelo. Megalitos, nudos en el espacio: Análisis...

misma práctica social e, incluso, por la misma comunidad. Al mismo tiempo el término ha sido confundido por las grandes necrópolis existentes en los poblados neolíticos y calcolíticos (piénsese, por ejemplo, en Los Millares).

De este modo, parecía lógico no utilizar ese término por cuanto su empleo supone determinadas presunciones sobre la realidad referida a través de él. Y sin embargo, ¿sería todavía posible hablar de "necrópolis" en un sentido positivo?

Tal y como ha sido puesto de manifiesto en múltiples ocasiones por V. Oliveira Jorge en A Aboboreira (1988), las mámoas que constituyen grupos importantes presentan un plan organizado que no parece resultado sin más del azar. Los túmulos se distribuyen ordenadamente utilizando la superficie disponible y emplazándose en aquellos puntos que sus condiciones de visibilidad requieren. Ahora bien, ese resultado no parece haber sido producido todo de una vez, no parece que su construcción sea simultánea y/o responda a un plan previo que se sigue de una forma rígida a través de un lapso de tiempo muy amplio. Antes bien habría que sospechar que cada nuevo monumento fue construido en donde le deja sitio el anterior, aunque procediendo de un modo organizado en vez de aleatorio. Este fenómeno se podría entrever a través de esas agrupaciones de túmulos en las que mientras unos túmulos se benefician de los emplazamientos más óptimos, otros intentan sacar el máximo partido posible del punto en el que se sitúan.

Aunque en la actual Galicia las grandes agrupaciones de mámoas no son un fenómeno dominante, tampoco predominan los casos de mámoas aisladas. Lo más frecuente es la aparición de túmulos agrupados en parejas o formando pequeños grupos de tres o cuatro ejemplares. En estos casos es asimismo normal que mientras un túmulo destaca por sus grandes proporciones y/o acentuadas condiciones de visibilidad, su/s compañero/s sean en cambio de pequeñas dimensiones y dispongan de una visibilización más restrictiva.

En el caso de la Sierra de O Bocelo, por ejemplo, de un total de 32 túmulos existentes en la zona estudiada, 20 están agrupados en siete parejas y dos triadas. Al mismo tiempo, en todos estos casos contrasta la asociación de un túmulo que puede llegar a los $30 \mathrm{~m}$ de diámetro y 2 $\mathrm{m}$ de altura y que, con independencia de que su visibilidad sea o bien general $o$ bien en abanico o linear, se diferencia desde considerable distancia, con otro túmulo de unos $16 \mathrm{~m}$ de diámetro, 0,5-1 $\mathrm{m}$ de altura y 


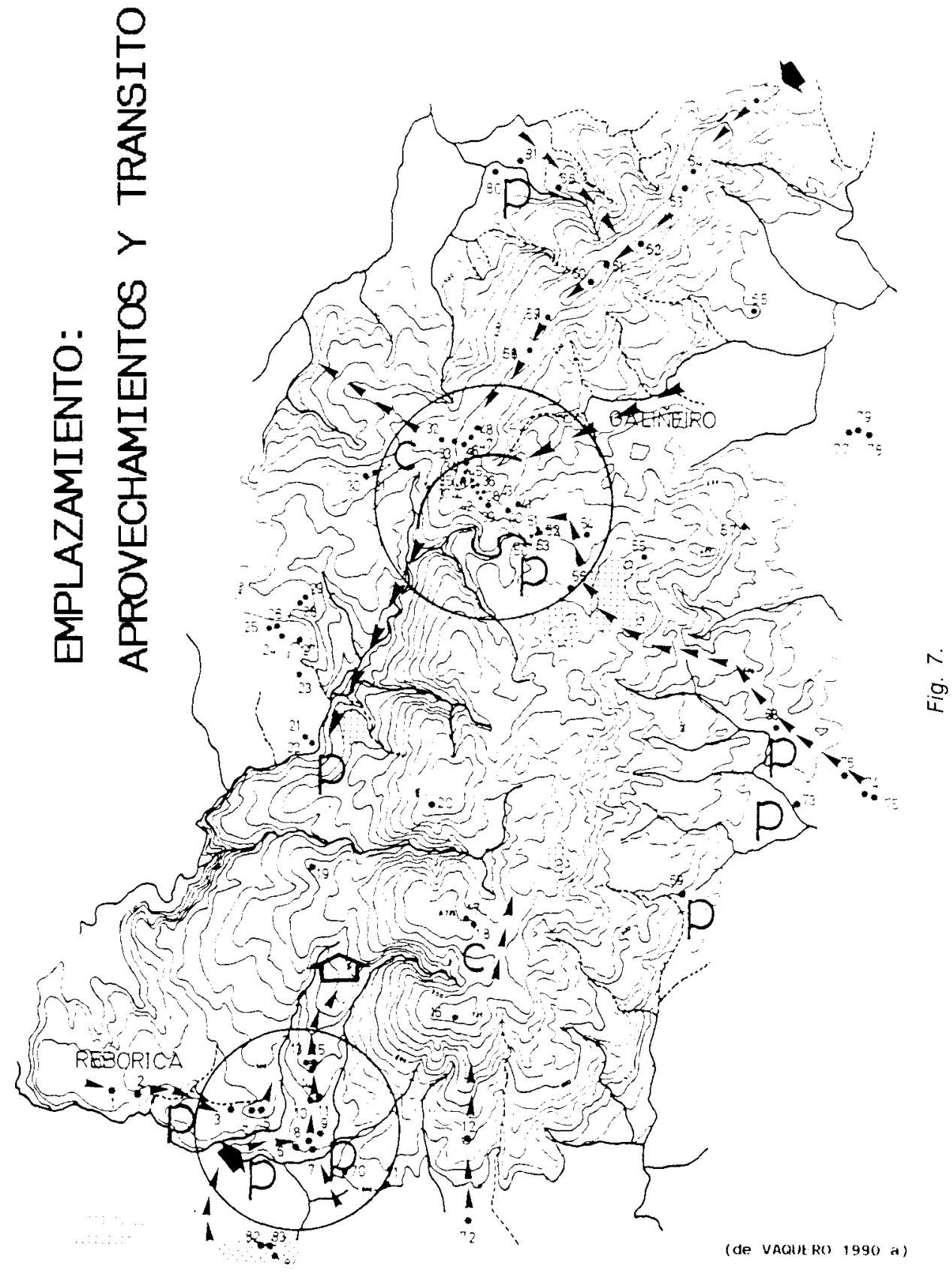




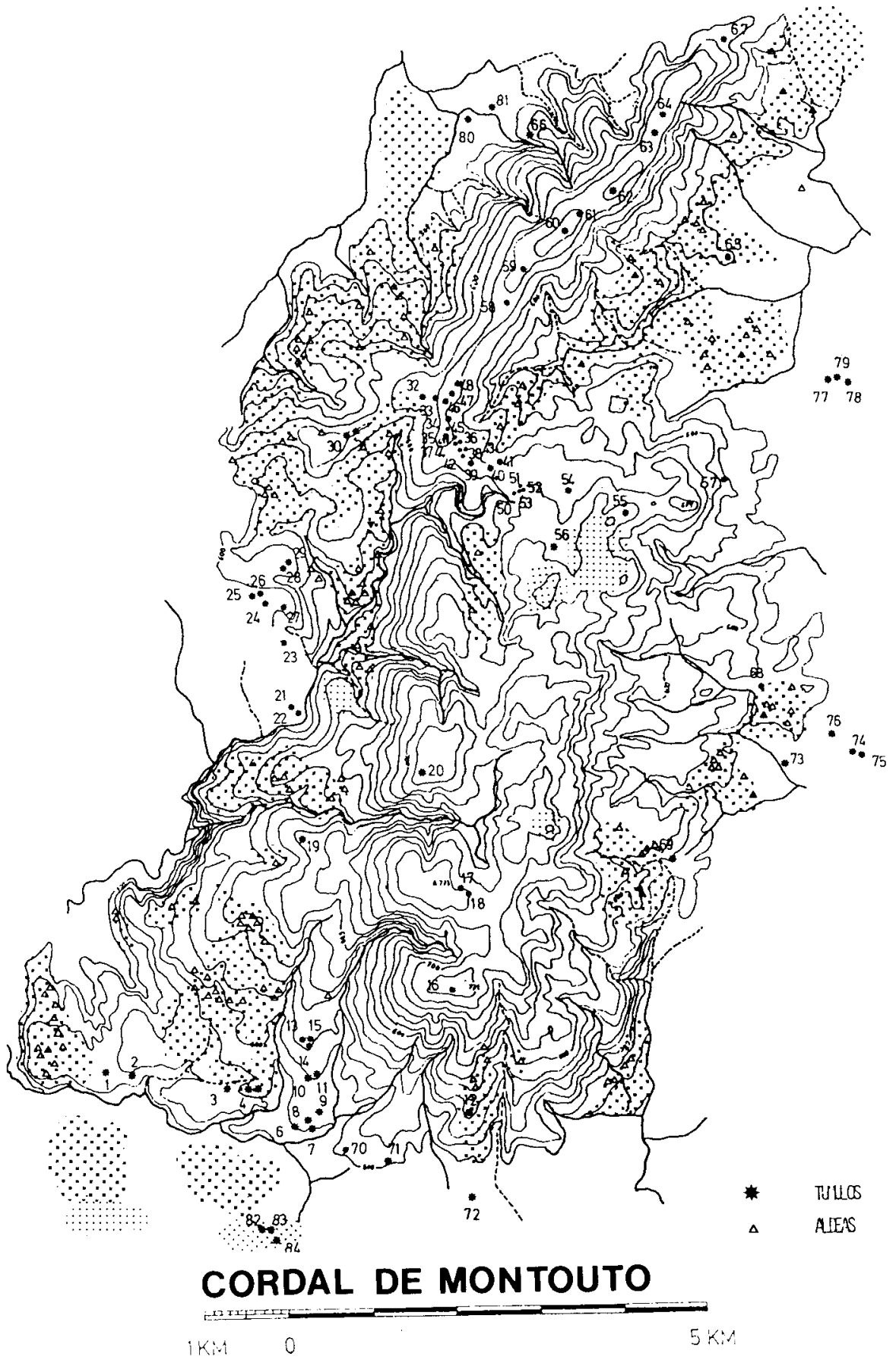

Fig. 7. 
una visibilidad puntual ${ }^{29}$. En algún caso, la visiblidad de esta segunda mámoa está inhibida por su vinculación a algún elemento topográfico o geológico que la oculta ${ }^{30}$.

En base a estos datos nos parece posible plantear la hipótesis de que esa disposición recurrente de un túmulo de determinadas características junto a otro de características distintas tal vez represente la intención consciente de emplazar un monumento tomando como factor dominante de vinculación la existencia de un monumento previo.

Este proceso sin duda oper'o de alguna forma en aquellas «provincias megalíticas" de la fachada atlántica en las que son frecuentes y dominantes los monumentos megalíticos construidos por la adición de nuevas cámaras a cámaras ya existentes ${ }^{31}$. En Galicia en cambio, en donde no disponemos de ese tipo de construcciones, la hipótesis anterior, podría venir avalada en parte por la documentación de que el conocido monumento megalítico de Dombate (Cabaña, Coruña) fue construido sobre un monumento anterior constituido por un túmulo más pequeño dotado de una reducida cámara megalítica ${ }^{32}$. Ante una «superposición» de este estilo, marcadamente anti-funcional, podemos aceptar que plantear la posibilidad de que el emplazamiento del segundo monumento intente beneficiarse del prestigio y cualidad simbólica inherente al primer monumento, tiene, al menos, visos de plausibidad.

Si dejamos nuestra zona de estudio por unos momentos, y miramos esta problemática desde el exterior, esa hipótesis no sólo se hace más plausible sino, en parte, también comprensible. R. BRADLEY (1986 y BRADLEY et al. 1988) ha destacado de qué modo los "cursi» del neolítico británico, construidos por primera vez hacia el 3300 a. C., han servido como focos de construcciones posteriores en sus alrededores, primero "henges" y después túmulos, durante el III milenio y principios de los II. Los "cursi» de Dorset y Stonehenge en el sur de Inglaterra, en torno a los cuales se desarrollaron auténticos complejos monumentales, son buenos ejemplos en este sentido. Este proceso de adición habría dado lugar a una especie de continuidad en el paisaje que, según este autor, se debe entender como un proceso de “invención de tradición». Ésta se basaría en la ca-

29 El único estudio publicado hasta el momento es CRIAdo y VaQuero 1990.

30 Así ocurre en el túmulo 21., situado al margen del gran túmulo que contiene el monumento megalítico de Forno dos Mouros (n. ${ }^{\circ}$ 20.).

Son los «multi-period tombs» de CORCARAN 1972

32 Este hecho, que está todavía inédito, ha sido descubierto por los trabajos realizados en ese monumento por nuestro compañero J.M. BELLo DiÉGUEZ, a quien agradecemos su autorización para utilizar esta referencia. 
Monumentos, nudos en el pañuelo. Megalitos, nudos en el espacio: Análisis...

pacidad de reconocer "cosas anteriores» que caracteriza a la experiencia del tiempo alcanzada en los rituales realizados alrededor de esos monumentos. De ese modo se construiría un pasado artificial que, de acuerdo con la argumentación de Bradley, podría ser una forma de apoyar la emergencia de élites sociales.

Incluso aunque no se acepte la totalidad de este argumento (con ramificaciones y consecuencias que aquí no hemos resumido), la idea de que la yuxtaposición de monumentos artificiales a lo largo de un decurso temporal amplio es un recurso convencional a través del cual se puede construir la imagen cultural de una tradición previa que preserva el presente social, es una sugerencia muy útil para interpretar la agrupación de mámoas en parejas o, en los casos más exarcebados, su adición paulatina hasta constituir grupos numerosos. En este sentido no sería incorrecto denominar a esas agrupaciones «necrópolis», entendiendo entonces con este concepto la vinculación de un túmulo a túmulos anteriores realizada con la intención de construir una apariencia de continuidad o una imagen de tradición ${ }^{33}$.

Pero con este desarrollo hemos hecho algo más que definir un concepto de un modo riguroso. Hemos descrito de qué forma la existencia de túmulos anteriores podría ser un factor de emplazamiento de los monumentos tumulares de la actual Galicia. Al mismo tiempo hemos apuntado el motivo posible que daría lugar a la formación de «necrópolis» en un contexto como el gallego en el que no existieron grandes aglomeraciones habitacionales en esa época ni, tal vez, una relación o continuidad directa entre todas las comunidades que levantaron los túmulos de una misma necrópolis.

La importancia de este nuevo factor de emplazamiento viene corroborada por la existencia de ciertos túmulos que no parecen estar vinculados a ninguno de los factores anteriores y cuyo emplazamiento semeja estar en función exclusivamente de la presencia de monumentos anteriores. En estos casos, las visibilidades entre mámoas definen un espacio más o menos "cerrado" al exterior que conforma el espacio significativo de la necrópolis, y de esta forma aquellas se convierten en el recurso que hace posible actualizar una imagen de «tradición».

Evidentemente ese espacio, cuando operan los otros tres factores de emplazamiento descritos, también estará abierto hacia afuera. En este

33 En realidad la frecuente «reutilización» por comunidades arqueológicas de monumentos o elementos anteriores tal vez se deba entender ante todo en este sentido. 
sentido es significativo el hecho de que las zonas que ocupan necrópolis importantes sean áreas que posean un privilegio especial, aunque no sea más que por disponer de una marcada visibilidad zonal. En algunos casos aquella zona de una comarca que más y mejor se ve desde las diferentes latitudes de esa comarca, es también la que concentra el mayor número de túmulos y en la que aparecen necrópolis muy extensas. Tal es el caso de la "Serra das Medoñas de Galiñeiro», entre los ayuntamientos de Aranga y Guitiriz, entre las provincias de Lugo y Coruña (VAQUERo LASTRES $1990 a$ y 1990 b) ${ }^{34}$.

No pretendemos ocultar que quedan muchos aspectos importantes pendientes. Entre ellos podemos resaltar sobre todo dos temas involucrados con el proceso de invención de la tradición.

El primero de ellos complementa y extiende la argumentación del apartado 3.2. En efecto, si allí hablábamos de la vinculación a accidentes geográficos significativos desde una perspectiva exclusivamente espacial, podríamos ahora introducir en la consideración de ese factor la perspectiva temporal. Pues si resulta que los hitos geográficos pueden formar un paisaje salvaje, no sería imposible que en un sentido muy lato la vinculación de los túmulos con esos hitos fuese también una forma de recoger y reintegrar una tradición pristina diluida casi en los contornos naturales del paisaje. Muchos túmulos se vinculan a ciertos elementos naturales que habrian poseído una significación especial, deciamos más arriba. Faltaba decir que esa relación habría operado entre los constructores de los túmulos. $Y$ con lo que ahora decimos queda abierta la posibilidad de que esa significación hubiera existido ya antes y que éstos últimos la recuperasen en forma de tradición.

El segundo tema, en vez de volver hacia atrás, mira hacia adelante y apunta fuera de los límites de este texto. Hablar de la invención de la “tradición» implica problemas de orden conceptual que no se pueden ocultar. Porque si se acepta que existe algo así como la tradición, entonces también se debe aceptar la existencia de una cierta concepción sobre el pasado, sobre el presente social e, incluso, sobre el futuro. $Y$ todo ello supone en último extremo asumir, no sólo que el «tiempo existe» dentro de esa sociedad que inventa la tradición, sino sobre todo que en ella se reconoce una determinada "configuración del tiempo". El problema que se alumbra aqui no es sólo de orden teórico, sino también práctico,

\footnotetext{
34 Sobre este fenómeno se pueden ver los casos enumerados en CRIADO. Fabregas et al. 1991 que, aunque sostienen una argumentación de carácter distinto a la que aquí nos ocupa, caen dentro de estas características.
} 
Monumentos, nudos en el pañuelo. Megalitos, nudos en el espacio: Análisis...

pues habría que considerar qué condiciones sociales y culturales acompañan a esa configuración del tiempo y posibilitan la invención de la tradición. En principio, la presencia de una tradición entendida y utilizada como fuerza positiva (que hace cosas) dentro de la sociedad, que implica un reconocimiento del pasado y que se circunscribe en un proyecto teleológico, parece ser una construcción propia de "sociedades campesinas". Sin embargo no es ésta ocasión de entendernos por esta línea que abre perspectivas muy vastas y de proyección diferente a la que aquí ahora se pretende; que redondea, en suma, la coherencia del megalitismo, pues si al principio veíamos que éste empezaba siendo un fenómeno de carácter espacial, al final, después de una descripción pormenorizada de algunas de sus características espaciales, vemos que termina siendo también un fenómeno de proyección temporal, una determinada maquinaria de construcción del tiempo.

En cualquier caso, hemos llegado a través de esta argumentación a un cuarto tipo de factores de emplazamiento de carácter totalmente cultural. Es un factor de emplazamiento que no tiene como referente una forma de paisaje ecológico ni una circunstancia del paisaje social. $Y$ por esa razón lo hemos definido como "escrito a pesar de la naturaleza».

\section{SINTTESIS: LA PREDICCIÓN DEL EMPLAZAMIENTO}

Al inicio de este trabajo dijimos que el emplazamiento está vinculado con procesos sociales. Pero al final del mismo, después de una redacción marcadamente descriptiva, no parece que hayamos avanzado demasiado en esa línea.

Sin embargo el objetivo de este trabajo no era fundamentalmente ése. Pues, dejando al margen que establecer esa relación entre práctica social y práctica ideacional megalítica debe ser objeto de un trabajo distinto, nuestra intención en este texto era mostrar que el emplazamiento, con el concurso del movimiento o por sí mismo, contribuye a hacer del monumento tumular una experiencia particular del tiempo y del espacio que contiene la racionalidad de lo que el megalitismo haya sido.

En este sentido esperamos que nuestro análisis pueda servir para continuar nuestra re-construcción actual de esa forma de hacer y pensar que una vez existió y que ya no existe, salvo transformaciones lejanas en versiones inesperadas.

Mientras las líneas fundamentales de ese proyecto mayor continúan afirmándose en otros trabajos en curso de realización, este texto se reduce 
en parte a la enunciación de un conjunto de hipótesis sobre el emplazamiento tumular.

Aunque no es fácil encontrar una forma objetiva de contrastación de esas hipótesis, también es cierto que si todo lo que hemos dicho sobre el ernplazamiento tumular es correcto, entonces se podría esperar que basándonos en ello pudiéramos «predecir la localización de túmulos» e, incluso, "prefigurar la situación de los hábitats" de sus constructores.

Lo primero se ha intentado hasta la fecha con cierta fortuna, aunque de una forma episódica, no sistemática y con finalidades fundamentalmente de carácter patrimonial ${ }^{35}$.

Lo segundo parece más difícil e interesante por cuanto en Galicia, al igual que en la mayor parte del megalitismo atlántico, no se conocen indicios de los hábitats de estos momentos. Sin embargo disponemos de algunos datos en este sentido que parecen apoyar o coincidir con las consideraciones que aquí hemos reunido. Para acabar este trabajo observaremos uno de los presumibles hábitats megalíticos localizados hasta la fecha y de este modo podremos finalizar además con una síntesis unitaria de todos los factores de emplazamiento descritos anteriormente.

Este ejemplo está tomado de un pequeño sector de la «Serra do Bocelo» (Toques, Coruña). En este punto se localizan una serie de túmulos dispuestos en la llanura superior de la sierra, sobre su divisoria, y a lo largo del "camiño real» que la atraviesa longitudinalmente. Entre estos túmulos se encuentra el monumento de “Forno dos Mouros», cámara poligonal con corredor largo y estructura de acceso a la misma compleja (posiblemente del tipo atrio). En la figura correspondiente se representan de forma esquemática la zona, las particularidades de la misma y la localización de los túmulos. En esa figura, además, intentamos resumir el efecto de los diferentes factores de emplazamiento que hemos descrito.

Factor 1. Los cuatro túmulos representados se agrupan en tres conjuntos. Todos ellos se alinean a lo largo de una vía longitudinal de tránsito (que definimos como "cuerda»). Dos mámoas, además, se emplazan en el cruce entre esta vía y otras vías transversales (en un collado concretamente).

Factor 2. Las mámoas 20. (Forno dos Mouros) y 21., se vinculan a una gran afloración granítica que posee unas marcadas características de

35 Los autores de este trabajo, por separado y conjuntamente, han hecho ensayos de predicción de la distribución de túmulos con el objeto de simplificar trabajos puntuales de prospección arqueológica. Los resultados preliminares son muy satisfactorios. 
Monumentos, nudos en el pañuelo. Megalitos, nudos en el espacio: Análisis...

visibilidad. La 21., además, queda parcialmente ocultada por esa afloración, pues la misma impide que se pueda diferenciar el túmulo desde el W.

Factor 3. Aunque los túmulos se disponen sobre zonas de cima de sierra, en sus inmediaciones el terreno se suaviza y se encuentran unas cubetas abrigadas en las que nacen las corrientes de agua de la zona y se generan pequeñas brañas. La zona en su conjunto es adecuada para el cultivo de cereal con sistema de rozas. En el tramo de pendiente anejo a una de estas cubetas, en una posición resguardada, orientada a mediodía y dominando una cuenca de condiciones favorables para su ocupación y utilización fue donde se encontró en el verano de 1989 un conjunto de materiales líticos y cerámicos que en principio parecen corresponder a momentos megalíticos ${ }^{36}$.

Factor 4. Las mámoas 20. y 21., presentan la dualidad de características que vimos anteriormente. La 21., queda ocultada por el $W$ por la afloración inmediata, como dijimos, y por el $\mathrm{S}$ por el túmulo 20 . Por el $\mathrm{N}$ y por el $\mathrm{E}$ apenas se puede ver porque, al caer pronunciadamente la pendiente, el ángulo de visión no permite observar la zona en la que sitúan. Pareciera que esta mámoa en parte oculta se vincula fundamentalmente a la afloración aneja y al túmulo 20 .

Como se puede observar (véase Figura 7), en este caso se aprecia la operación conjunta de todos los factores definidos. La coherencia de este ejemplo viene destacada, además, por la aparición del presente hábitat megalítico. Éste muestra una relación visual muy clara con los túmulos inmediatos, pues desde la posición que ocupa se diferencian perfectamente recortándose sobre el terreno circundante los túmulos 20 . , 21. y 22. El túmulo 21., se percibe a pesar de las dificultades de visibilización que acabamos de citar, y el 22., a pesar de encontrarse en un angosto collado que hace que posea una visibilidad de tipo lineal muy restringida.

En cualquier caso esta investigación no está finalizada. Este texto presenta el modelo inicial de emplazamiento tumular que deberá ser confirmado en ulteriores análisis. Para continuar con éstos, la mayor dificultad radica en encontrar un sistema de análisis emancipado de apreciaciones subjetivas.

36 Los datos ergológicos se pueden consultar en MENDEZ González 1990, y los datos locacionales en ese mismo trabajo y en Criado y VAquero 1990. 


\section{BIBLIOGRAFIA}

Amor Meilán. A. (1918): Historia de Lugo. Coruña.

BARRETT. J. C. (1983): Goodbye, New Archaeology?, Current Archaeology VIII (6), págs. 188-9.

Barros Silvelo, M. 1875: Antigüedades de Galicia.

Barthes, R. (1980): Mitologias. Madrid: Siglo XXI, (París: Seuil, 2. ${ }^{a}$ edic. 1980. -1. a edic. $1957-)$.

BÁs. B. (1985): “Función e significado das pontes tradicionais", Actas do Congreso a inxeñería histórica como patrimonio monumental: as pontes, págs. 1-10. Santiago.

Bello Diéguez. J. M., Criado Boado. F. y Vazquez Varela. J. M. (1982): "Sobre la cultura megalítica y los caminos antiguos en Galicia", Museo de Pontevedra XXXVI, págs. 143-64.

- (1984): "Medio físico y sociedades megalíticas. Aproximaciones a los problemas constructivos de los megalitos en el NW peninsular", Gallaecia 7-8, págs. 31-58.

BraDLEY, R. (1986): The Dorset cursus: the archaeology of the enigmatic. Wessex lecture III (London: $\mathrm{CBA}$ ).

Bradley, R. y Chambers. R. (1988): "A new study of the cursus complex at Dorchester on Thames", Oxford Journal of Archaelogy 20, págs. 249-60.

Carlos IzQUiERdo, J. I. DE (1988): “Una aproximación territorial al fenómeno megalítico: la Rioja Alavesa y Cuartango", Munibe suplemento n. ${ }^{\circ} 6$, págs. 113-27

Cerqueiro Landin. D. (1989): La utilización del espacio en el Paleolítico: patrón de asentamiento en la Sierra de O Bocelo. Santiago, Facultade de Xeografía e Historia, (Trabajo de Investigación del Tercer Ciclo, inédito)

- (1990): "Industrias líticas: un pasado difuso", en CrIADO, F., Bonilla, A. et al. en prensa.

Chalmers. A. F. (1990): ¿Qué es esa cosa llamada ciencia? Una valoración de la naturaleza y el estatuo de la ciencia y sus métodos. Madrid, Siglo XXI, (8. ${ }^{2}$ edic., la edic. 1982; University of Queensland Press, 1982 -2. ${ }^{\text {a }}$ edic. corregida-, 1976 -la edic. $\longrightarrow$ ).

Chapman, R. W. (1987): «Megalitisme i Arqueologia: problems, teoria i investigació, Cota Zero 3, págs. 93-102.

CLARKE. D. L. (ed.) (1977): Spatial Archaeology. London, Academic Press.

Clastres, P. (1981): Investigaciones en Antropologia Politica. Barcelona, Gedisa, (Paris, Seuil, 1980).

CoRCoran, J. X. W. (1972): "Multi-period construction and the origins of the chambered long cairn in Western Britain and Ireland", en F. LYNCH y C. BuRGUES (eds.) Prehistoric man in Wales and the West, págs, 31-64. Bath.

CRIADO BOADO, F. (1984-5): “El tercer factor» o la lógica oculta del emplazamiento de los túmulos megalíticos gallegos", Cuadernos de Estudios Gallegos XXXV (100), págs. 7-18.

- (1988): “Arqueología del Paisaje y Espacio Megalítico en Galicia", Arqueologia Espacial 12. págs. 61-117. Teruel, Colegio Universitario de Teruel.

- (1989a): "Megalitos, Espacio, Pensamiento", Tranbajos de Prehistoria 46, págs. 75-98.

- (1989b): “Asentamiento Megalítico y Asentamiento Castreño: una propuesta de síntesis", Gallaecia 11, págs. 109-37.

- (1989c): "We, the post-megalithic people...", en 1. Hodder (ed.) The Meanning of Things. Material Culture and Symbolic Expression, págs. 79-89. London, Unwin Hyman.

- (1991): "Más allá de la Arqueología Espacial. Sociedades, Paisajes, Pensamientos", Boletín de Antropologia Americana, en prensa. México.

Criado Boado. F. y Fabregas Valcarce. R. (1989): “Main trends in the Megalithic Pehenomenon of NW Spain", Antiquity 63 (241), págs. 682-96.

Criado Boado, F. y Penedo Romero, R. (1989): "Arte de cazadores y arte de salvajes: una contraposición entre el arte paleolítico y el arte post-glaciar levantino», Munibe 41, págs. 3 22.

Criado Boado. F. y Vaquero Lastres, X. (1990): "Formas diversas de pasado monumental", en Caiado, F., Bonilla. A. et al., en prensa.

Criado Boado. F., Aira Rodríguez. M. J. y Diaz-Fierros Viqueira. F. (1986): La construcción del paisaje. Megalitismo y Ecologia en la Sierra de Barbanza. Santiago, Xunta de Galicia, Dirección Xeral do Patrimonio Artístico e Monumental. 
Monumentos, nudos en el pañuelo. Megalitos, nudos en el espacio: Análisis...

Chiado Boado, F., fabregas Valcarce, R. y Vaquero lastres, X. (1991); "Concentraciones de túmulos y vías naturales de acceso al interior de Galicia", Portugalia, en prensa. Porto.

Criado Boado. F., Bonilla Rodriguez. A., Cerqueiro landín. D., González Méndez. M., Méndez Fernandez y Penedo Romero. R. (1989): "Proyecto de Prospección Intensiva en la Sierra de O Bocelo y en el valle del río Furelos, (A Coruña)", Sintesis del Informe Preliminar de la Campaña de 1987. Arqueoloxía-Informes (Campaña de 1987) 1, págs. 128-34. Santiago, Consellería de Cultura, Xunta de Galicia.

Criado Boado. F., Bonilla Rodriguez, A., Ceroueiro landín. D., infante Roura, F., Gonzalez Méndez, M., Mendez fernandez. Penedo Romero. R., Rodriguez Puentes. E., Vaquero Lastres. J. y Vázouez Díaz. M. (1990a): Arqueologia del Paisaje en Galicia. El área BoceloFurelos desde el Paleolítico Superior Final hasta la Época Medieval. (Campañas de 1987, 1988 y 1989, Arqueoloxia-Investigación, 6. Santiago, Xunta de Galicia, Dirección Xeral do Patrimonio Histórico, en prensa.

- (1990b): "Arqueología del Paisaje. Actuaciones arqueológicas en la sierra de O Bocelo y en el valle del río Furelos, (Melide-Toques, Coruña), Informe Preliminar de la Campaña de 1988". Arqueoloxia-Informes (Campaña de 1988) 2, págs. 101-30. Santiago, Conselleria de Cultura, Xunta de Galicia.

Cruz. D. J. da (1988): "O Megalitismo do Norte de Portugal", en Coloquio de Arqueologia do NW Peninsular. Porto 1988, págs. 15-50. Trabalhos de Antropologia e Etnologia, vol. XXVIII.

Diaz Sanjurjo. M. (1902-6): "Los caminos antiguos en el itinerario n. ${ }^{\circ} 18$ de Antonino en la provincia de Orense", Bol. Comis. Monumentos de Orense, II-III.

FABREgAS VALCARCE, R. (1990): Estudio de los ajuares liticos de las sepulturas megaliticas de Galicia y norte de Portugal. Madrid: Universidad a Distancia, (edic. en microficha).

FLEMING, A. (1973): “Tombs for the livings", Man VIII, págs. 177-93.

Germont, A. (1980): Inventaire des megalithes de la France. Gallia Prehistoire, suppl. 6. Paris.

Hodder. I. (1989): "Post-modernism, Post-structuralism and Post-processual archaeology", en I. Hodder (ed.) The Meaning of Things. Material Culture and Symbolic Expression, págs 64 78. London, Unwin Hyman

Infante Roura, F., Vaduero Lastaes, J. y Criado Boado, F. (1990): "Vacas, caballos, abrigos y túmulos: definición de la geografía del movimiento para el estudio arqueológico", Cuadernos de Estudos Galegos, en prensa.

L'HeLgouach, J. (1965): Les sepultures megalithiques en Armorique. Rennes, Tésis Doctoral.

LEISNER. G. (1938): Verbreitung und Typologie der Galizisch-Nordportugiesischen Megalithgräber. Marburg.

Lévi-Strauss, Cl. (1964): El pensamiento salvaje. México, Fondo de Cultura Económica, (tercera reimpresión, 1975. París, Plon, 1962).

LEVINE. P. (1986): The amateur and the professional. Antiquarians, Historians and Archaeologists in Victorian England, 1838-1886. Cambridge, Cambridge University Press.

LOPEz Cuevillas. F. (1925): "As mámoa do concello de Rairiz", Bol. Real Academia Gallega 15, págs. $235-45$.

- (1979): “A edade megalitica», en R. Otero Pedrayo (dir.) Historia de Galiza, págs. 43-122. Madrid, Akal, (edic. facsímil, edic. orig. Buenos Aires 1952.

MACINEIRA y PARDO DE LAMA. F. (1943-4): "Túmulos prehistóricos. Inventario descriptivo de los 286 túmulos prehistóricos hasta ahora descubiertos en la avanzada comarca del cabo Ortegal", Boletin de la Real Academia Gallega XXIII y XXIV, págs. 21-9, 1.124-35, 178-86, 236-9 y 314-21, págs. 15-34.

- (1947): Bares, puerto hispánico de la primitiva navegación occidental. Madrid, CSIC.

Madariaga, B. y SaUtuola, M. (eds.) (1976): Marcelino Sanz de Sautuola. Escritos y documentos. Santander, Institución Cultural de Cantabria.

MÉndez GonzÁlez. M. (1990): "Yacimientos del III Milenio a.C.: entre la problemática del calcolítico y un pasado huidizo", en F. CRiAdo, A., Bonilla, et al., en prensa.

RENFREW, C. (1973): "Monuments, mobilisation and sociall organization in neolithic Wessex", en RENFREW. C. (ed.) The explanation of culture change: models in prehistory, págs. 539-58. London, Duckworth.

- (1976): "Megaliths, Territoires and Populations", en S. J. de Laet (ed.), Acculturation and Continuity in At/antic Europe, págs. 198-220. Dissertations Archaeologicae Gandenses, XVI. 
- (1987): «El arqueólogo y su pensamiento. Una conversación con C. Renfrew», (Entrevista realizada por F. Criado, y C. Damm). Revista de Arqueología 78, 5-13.

Rodriguez CASal. A. (1983): “A mámoa de Monte Campelos e o seu enterramento secundario. Novos datos encol do Megalitico final galego", Boletin do Museo Provincial de Lugo I, págs. 7-17.

Shanks, M., y Tilley, C. (1987a): Re-constructing archaeology. Cambridge, Cambridge University Press.

TiLLEY, C. (1989): "Interpreting material culture", en I. Hodder (ed.) The Meaning of Things. Material Culture and Symbolic Expression, págs. 185-94. London, Unwin Hyman.

VAQuero LASTRES. J. (1989a): “¿Dónde diablos se esconden nuestros muertos que no los podemos ver? Reflexiones sobre el emplazamiento de los túmulos del NW", Gallaeica 11, págs. $81-108$.

- (1989b): "Prospección en Serra da Loba e Cordal de Montouto: el fenómeno tumular", Brigantium 6, en prensa.

- (1990a): El fenómeno tumular en el interior de Galicia: consideraciones sobre el emplazamiento de los monumentos. Santiago, Fac. Xeografía e Historia, (tesis de licenciatura inédita).

- (1990b): "Galiñeiro, paso de novios, lobos y héroes", Cuadernos de Estudios Gallegos, en prensa.

(1990c): "Rios y Tumbas. Sobre el emplazamiento de túmulos en el NW peninsular", Trabalhos de Antropologia e Etnologia 30, 151-75. 